\title{
QUANTUM GROUPS ACTING ON 4 POINTS
}

\author{
TEODOR BANICA AND JULIEN BICHON
}

\begin{abstract}
We classify the compact quantum groups acting on 4 points. These are the quantum subgroups of the quantum permutation group $\mathcal{Q}_{4}$. Our main tool is a new presentation for the algebra $\mathrm{C}\left(\mathcal{Q}_{4}\right)$, corresponding to an isomorphism of type $\mathcal{Q}_{4} \simeq S O_{-1}(3)$. The quantum subgroups of $\mathcal{Q}_{4}$ are subject to a McKay type correspondence, that we describe at the level of algebraic invariants.
\end{abstract}

\section{INTRODUCTION}

After the seminal work of Drinfeld [19] and Woronowicz [42], an influencal treatment in quantum group theory was Manin's book [28]. Manin proposed to construct quantum groups as quantum symmetry groups of quantum spaces, which were seen there as dual objects of quadratic algebras. Later on, Wang [41] studied quantum symmetry groups of finite quantum spaces, corresponding to finite-dimensional $\mathrm{C}^{*}$-algebras, answering in this way a question of Connes on the existence of such quantum groups. One surprising conclusion of Wang's work is that there exist infinite compact quantum groups acting faithfully on $n$ points, provided $n \geq 4$. The biggest such quantum group, denoted here by $\mathcal{Q}_{n}$, is called the quantum permutation group on $n$ points. In other words, the symmetric group $S_{n}$ has $\mathcal{Q}_{n}$ as an infinite quantum analogue if $n \geq 4$.

Soon after Wang's discovery, the representation theory of the quantum group $\mathcal{Q}_{n}$ was worked out in [2]: the fusion semiring is identical to the one of the compact group $S O(3)$ if $n \geq 4$. A very rough explanation for this result is the fact that $S O(3)$ is the (quantum) automorphism group of the $\mathrm{C}^{*}$-algebra $M_{2}(\mathbb{C})$, and hence $S O(3)$ and $\mathcal{Q}_{n}$ have the same kind of universal property.

Since then work on quantum permutation groups has been done mainly in two directions. The first one is the construction of non-classical quantum permutation groups, i.e. quantum subgroups of $\mathcal{Q}_{n}$, using finite graphs $[14,15,4,5,6,7]$. The other direction is the study of the structure of the $\mathrm{C}^{*}$-algebra $\mathrm{C}\left(\mathcal{Q}_{n}\right)$. A general matrix representation was constructed in [12], which, using the explicit description of the Haar measure [10,11], was shown to be faithful at $n=4$, giving an embedding $\mathrm{C}\left(\mathcal{Q}_{4}\right) \subset M_{4}(\mathrm{C}(S U(2)))$ [11]. The case $n \geq 5$ is certainly much harder to understand, because of the non-amenability of the discrete quantum group dual to $\mathcal{Q}_{n}[2]$.

A natural problem in the area is classification problem for quantum permutation groups on $n$ points, at least for small $n$. This paper deals with the case $n=4$.

2000 Mathematics Subject Classification. 20G42,16W30.

Key words and phrases. Quantum permutation group. 
The first result is a new presentation for the algebra $\mathrm{C}\left(\mathcal{Q}_{4}\right)$. This shows that $\mathcal{Q}_{4}$ is in fact isomorphic with $S O_{-1}(3)$, a $q$-analogue of $S O(3)$ at $q=-1$. Here a few comments are in order: $q$-deformations of classical groups have been defined by many authors at different levels of generality (see e.g. the book [26]) but we have never seen a non-trivial compact quantum group $S O(3)$ at $q=-1$. For example the quantum $S O_{-1}(3)$ in [34] is just the classical $S O(3)$. However it would be difficult to claim that the quantum $S_{-1}(3)$ found here is new: the algebra $\mathrm{C}\left(S O_{-1}(3)\right)$ is just the quotient of $\mathrm{C}\left(S U_{-1}(3)\right)$ by the relations making the fundamental matrix orthogonal.

The very first consequence of the presentation result is that all the irreducible $*$ representations of the $\mathrm{C}^{*}$-algebra $\mathrm{C}\left(\mathcal{Q}_{4}\right)$ are finite dimensional, and have dimension 1,2 or 4 . Then we show that $\mathrm{C}\left(\mathcal{Q}_{4}\right)$ is a deformation of $\mathrm{C}(S O(3))$ by an appropriate 2-cocycle. This is used in an essential way for the classification result, which is as follows.

Theorem 1.1. The compact quantum subgroups of $\mathcal{Q}_{4}$ are exactly, up to isomorphism, the following ones.

(1) $\mathcal{Q}_{4} \simeq S O_{-1}(3)$.

(2) The quantum orthogonal group $O_{-1}(2)$.

(3) $\widehat{D}_{\infty}$, the quantum dual of the infinite dihedral group.

(4) The symmetric group $S_{4}$ and its subgroups.

(5) The quantum group $S_{4}^{\tau}$, the unique non-trivial twist of $S_{4}$.

(6) The quantum group $A_{5}^{\tau}$, the unique non-trivial twist of the alternating group $A_{5}$.

(7) The quantum group $D_{n}^{\tau}, n$ even and $n \geq 6$, the unique non-trivial twist of the dihedral group of order $2 n$.

(8) The quantum group $D C_{n}^{\tau}$ of order $4 n, n \geq 2$, a pseudo-twist of the dicyclic group of order $4 n$.

(9) The quantum group $\widehat{D}_{n}, n \geq 3$, the quantum dual of the dihedral group of order $2 n$.

All the quantum groups appearing in the classification are already known. The quantum group $O_{-1}(2)$ was constructed in [15] as the quantum automorphism group of the graph formed by two segments. The twisted quantum groups $S_{4}^{\tau}$ and $A_{5}^{\tau}$ were constructed by Nikshych in [33]. The twisted quantum groups $D_{n}^{\tau}$ ( $n$ even and $n \geq 6)$ and pseudo-twisted $D C_{n}^{\tau}(n \geq 2)$ appear in papers by Nikshych [33] and Vainerman [38], or independently in Suzuki's paper [37] or Masuoka's paper [28]. Note that $D C_{n}^{\tau}$ is also a pseudo-twist of $D_{2 n}$, because they have the same fusion semiring, but we prefer to refer to the dicyclic group because of the constructions in $[33,28]$. At $n=2$ the quantum group $D C_{2}^{\tau}$ is the one corresponding to the 8-dimensional historical Kac-Paljutkin example of a non-trivial Hopf algebra. On the other hand, the occurence of $A_{5}^{\tau}, D_{n}^{\tau}$ and $D C_{n}^{\tau}$ as quantum permutation groups of 4 points seems to be new.

The classification for finite quantum groups also uses in an essential way fundamental results of Etingof and Gelaki [21, 22, 23].

The quantum groups in the above list can be arranged in an ADE table, by using the McKay correspondence and various techniques from [9]. 
The paper is organized as follows: 2 is a preliminary section, in 3-7 we prove the main result, and in 8-11 we write down ADE classification tables. The final section, 12 , contains a few concluding remarks.

\section{Compact quantum groups}

We first recall some basic facts concerning compact quantum groups and quantum permutation groups. The book [26] is a convenient reference for the topic of compact quantum groups, and all the possibly missing definitions might be found there. All the algebras are unital, and $\otimes$ denotes the minimal tensor product of $\mathrm{C}^{*}$-algebras as well as the algebraic tensor product: this should cause no confusion.

Definition 2.1. A Woronowicz algebra is a $\mathrm{C}^{*}$-algebra $A$ endowed with a *morphism $\Delta: A \rightarrow A \otimes A$ satisfying the coassociativity condition and the cancellation law

$$
\overline{\Delta(A)(A \otimes 1)}=A \otimes A=\overline{\Delta(A)(1 \otimes A)}
$$

The morphism $\Delta$ is called the comultiplication of $A$.

The category of Woronowicz algebras is defined in the obvious way (see [40] for details). A commutative Woronowicz algebra is necessarily isomorphic with $\mathrm{C}(G)$, the function algebra on a unique compact group $G$, and the category of compact quantum groups is defined to be the category dual to the category of Woronowicz algebras. Hence to any Woronowicz algebra $A$ corresponds a unique compact quantum group according to the heuristic formula $A=\mathrm{C}(G)$. Also to $A$ corresponds a unique discrete quantum group $\Gamma$, with $A=\mathrm{C}^{*}(\Gamma)$. The quantum groups $G$ and $\Gamma$ are dual of each other. Summarizing, we have

$$
\begin{gathered}
A=\mathrm{C}(G)=\mathrm{C}^{*}(\Gamma) \\
\Gamma=\widehat{G}, \quad G=\widehat{\Gamma}
\end{gathered}
$$

Woronowicz's original definition for matrix compact quantum groups [42] is still the most useful in concrete situation, and we have the following fundamental result [44].

Theorem 2.1. Let $A$ be a $\mathrm{C}^{*}$-algebra endowed with a *-morphism $\Delta: A \rightarrow A \otimes A$. Then $A$ is a Woronowicz algebra if and only if there exists a family of unitary matrices $\left(u^{\lambda}\right)_{\lambda \in \Lambda} \in M_{d_{\lambda}}(A)$ satisfying the following three conditions.

(1) The subalgebra $A_{0}$ generated by the entries $\left(u_{i j}^{\lambda}\right)$ of the matrices $\left(u^{\lambda}\right)_{\lambda \in \Lambda}$ is dense in $A$.

(2) For $\lambda \in \Lambda$ and $i, j \in\left\{1, \ldots, d_{\lambda}\right\}$, one has $\Delta\left(u_{i j}^{\lambda}\right)=\sum_{k=1}^{d_{\lambda}} u_{i k}^{\lambda} \otimes u_{k j}^{\lambda}$.

(3) For $\lambda \in \Lambda$, the transpose matrix $\left(u^{\lambda}\right)^{t}$ is invertible.

In fact the $*$-algebra $A_{0}$ in the theorem is canonically defined, and is what is now called a CQG algebra: a Hopf $*$-algebra having all its finite-dimensional comodules equivalent to unitary ones (see [26] for details). The counit and antipode of $A_{0}$, denoted respectively $\varepsilon$ and $S$, are refered as the counit and antipode of $A$. The Hopf algebra $A_{0}$ is called the algebra of representative functions on the compact quantum group $G$ dual to $A$, with another heuristic formula

$$
A_{0}=\mathcal{R}(G)=\mathbb{C}[\Gamma]
$$


Conversely, starting from a CQG-algebra, the universal $\mathrm{C}^{*}$-completion yields a Woronowicz algebra in the above sense: see the book [26]. In fact there are possibly several different $\mathrm{C}^{*}$-norms on $A_{0}$, but we will not be concerned with that problem.

As usual, a (compact) quantum subgroup $H \subset G$ corresponds to a Woronowicz algebra surjective morphism $\mathrm{C}(G) \rightarrow \mathrm{C}(H)$, or to a surjective Hopf $*$-algebra morphism $\mathcal{R}(G) \rightarrow \mathcal{R}(H)$.

We have the following key examples, due to Wang (respectively in [40] and [41]). First we need some terminology. A matrix $u \in M_{n}(A)$ is said to be orthogonal if $u=\bar{u}$ and $u u^{t}=I_{n}=u^{t} u$. A matrix $u$ is said to be a magic unitary if all its entries are projections, all distinct elements of a same row or same column are orthogonal, and sums of rows and columns are equal to 1. A magic unitary matrix is orthogonal.

Definition 2.2. The $\mathrm{C}^{*}$-algebras $A_{o}(n)$ and $A_{s}(n)$ are constructed as follows.

(1) $A_{o}(n)$ is the universal $\mathrm{C}^{*}$-algebra generated by variables $\left(u_{i j}\right)_{1 \leq i, j \leq n}$, with relations making $u=\left(u_{i j}\right)$ an orthogonal matrix.

(2) $A_{s}(n)$ is the universal $\mathrm{C}^{*}$-algebra generated by variables $\left(u_{i j}\right)_{1 \leq i, j \leq n}$, with relations making $u=\left(u_{i j}\right)$ a magic unitary matrix.

The $\mathrm{C}^{*}$-algebras $A_{o}(n)$ and $A_{s}(n)$ are Woronowicz algebras, with comultiplication, counit and antipode defined by

$$
\Delta\left(u_{i j}\right)=\sum_{k} u_{i k} \otimes u_{k j}, \varepsilon\left(u_{i j}\right)=\delta_{i j}, S\left(u_{i j}\right)=u_{j i}^{*}
$$

We now come to quantum group actions, studied e.g. in [34]. They correspond to Woronowicz algebra coactions.

Definition 2.3. Let $B$ be a $\mathrm{C}^{*}$-algebra. A (right) coaction of a Woronowicz algebra $A$ on $B$ is a $*$-homomorphism $\alpha: B \rightarrow B \otimes A$ satisfying the coassociativity condition and

$$
\overline{\alpha(B)(1 \otimes A)}=B \otimes A
$$

Wang has studied quantum groups actions on finite-dimensional $\mathrm{C}^{*}$-algebras in [41], where the following result is proved.

Theorem 2.2. The algebra $A_{s}(n)$ has the following properties.

(1) It is the universal Woronowicz algebra coacting on $\mathbb{C}^{n}$.

(2) It is infinite-dimensional if $n \geq 4$.

The coaction is constructed in the following manner. Let $e_{1}, \ldots, e_{n}$ be the canonical basis of $\mathbb{C}^{n}$. Then the coaction $\alpha: \mathbb{C}^{n} \rightarrow \mathbb{C}^{n} \otimes A_{s}(n)$ is defined by the formula

$$
\alpha\left(e_{i}\right)=\sum_{j} e_{j} \otimes u_{j i}
$$

We refer the reader to [41] for the precise meaning of universality in the theorem, but roughly speaking this means that the quantum group corresponding to $A_{s}(n)$, denoted $\mathcal{Q}_{n}$ (and hence $A_{s}(n)=\mathrm{C}\left(\mathcal{Q}_{n}\right)$ ), is the biggest one acting on $n$ points, and deserves to be called the quantum permutation group on $n$ points.

Equivalently, Wang's theorem states that any Woronowicz algebra coacting faithfully on $\mathbb{C}^{n}$ is a quotient of the Woronowicz algebra $A_{s}(n)$, and shows that quantum groups acting on $n$ points (quantum permutation groups) correspond to 
Woronowicz algebra quotients of $A_{s}(n)$. In particular there is a Woronowicz algebra surjective morphism $A_{s}(n) \rightarrow \mathrm{C}\left(S_{n}\right)$, yielding a quantum group embedding $S_{n} \subset \mathcal{Q}_{n}$. More directly, the existence of the surjective morphism $A_{s}(n) \rightarrow \mathrm{C}\left(S_{n}\right)$ follows from the fact that $\mathrm{C}\left(S_{n}\right)$ is the universal commutative $\mathrm{C}^{*}$-algebra generated by the entries of a magic unitary matrix. See [41] for details.

At $n=4$, by the (co)amenability result of [2], the study of the (compact) quantum subgroups of $\mathcal{Q}_{4}$ reduces to the study of the CQG algebra quotients of $A_{s}(4)_{0}=$ $\mathcal{R}\left(\mathcal{Q}_{4}\right)$.

\section{The QuAntum Symmetry group of 4 POINTS}

In this section we give a new presentation for the $\mathrm{C}^{*}$-algebra $A_{s}(4)$. The basic idea is to use an appropriate basis of the algebra $\mathbb{C}^{4}$. In general the algebra $\mathbb{C}^{n}$ is seen as the function algebra on the cyclic group $\mathbb{Z}_{n}$, and the Fourier transform yields an appropriate basis for the study of many quantum permutation groups [4]. Here the idea is to see $\mathbb{C}^{4}$ as the function algebra on the Klein group $\mathbb{Z}_{2} \times \mathbb{Z}_{2}$, and to use the Fourier transform of this group.

We will show that $A_{s}(4)$ is isomorphic with the following Woronowicz algebra.

Definition 3.1. The $\mathrm{C}^{*}$-algebra $\mathrm{C}\left(\mathrm{SO}_{-1}(3)\right)$ is the $\mathrm{C}^{*}$-algebra presented by generators $\left(a_{i j}\right)_{1 \leq i, j \leq 3}$ and submitted to the following relations.

(1) The matrix $a=\left(a_{i j}\right)$ is orthogonal,

(2) $a_{i j} a_{i k}=-a_{i k} a_{i j}$ and $a_{j i} a_{j k}=-a_{j k} a_{j i}$, for $j \neq k$,

(3) $a_{i j} a_{k l}=a_{k l} a_{i j}$, for $i \neq k$ and $j \neq l$,

(4) $\sum_{\sigma \in S_{3}} a_{1 \sigma(1)} a_{2 \sigma(2)} a_{3 \sigma(3)}=1$.

It is immediate to check that $\mathrm{C}\left(S O_{-1}(3)\right)$ is a Woronowicz algebra with

$$
\Delta\left(a_{i j}\right)=\sum_{k} a_{i k} \otimes a_{k j}, \varepsilon\left(a_{i j}\right)=\delta_{i j}, S\left(a_{i j}\right)=a_{j i}
$$

The last three families of relations show that, using Rosso's presentation for the quantum group $S U_{-1}(N)$ [35], there is a surjective Woronowicz $\mathrm{C}^{*}$-algebra morphism

$$
\mathrm{C}\left(S_{-1}(3)\right) \rightarrow \mathrm{C}\left(S O_{-1}(3)\right)
$$

The following notation will be convenient. For $i, j \in\{1,2,3\}$ with $i \neq j$, let $\langle i, j\rangle$ be the unique element in $\{1,2,3\}$ such that $\{i, j,\langle i, j\rangle\}=\{1,2,3\}$.

Lemma 3.1. Let $i, j, k, l \in\{1,2,3\}$ with $i \neq j$ and $k \neq l$. We have

$$
a_{\langle i, j\rangle\langle k, l\rangle}=a_{i k} a_{j l}+a_{j k} a_{i l}
$$

Proof. For $i, j \in\{1,2,3\}$, let $i_{1}, i_{2}, j_{1}, j_{2}$ be the elements of $\{1,2,3\}$ satisfying $\left\{i, i_{1}, i_{2}\right\}=\{1,2,3\}=\left\{j, j_{1}, j_{2}\right\}$. The well-known formula for the antipode in $\mathrm{C}\left(S U_{-1}(3)\right)$ gives

$$
S\left(a_{j i}\right)=a_{i_{1} j_{1}} a_{i_{2} j_{2}}+a_{i_{2} j_{1}} a_{i_{1} j_{2}}
$$

But since the matrix $a$ is orthogonal, we have $a_{i j}=S\left(a_{i j}\right)$, which proves the statement.

We now prove the presentation result.

Theorem 3.1. We have a Woronowicz algebra isomorphism $\mathrm{C}\left(\mathcal{Q}_{4}\right) \simeq \mathrm{C}\left(\mathrm{SO}_{-1}(3)\right)$. 
Proof. Let us show that $\mathrm{C}\left(S_{-1}(3)\right)$ is the universal Woronowicz algebra coacting on $\mathbb{C}^{4}$. Let $e_{1}, e_{2}, e_{3}, e_{4}$ be the canonical basis of $\mathbb{C}^{4}$. We use the following basis

$$
\begin{aligned}
1 & =e_{1}+e_{2}+e_{3}+e_{4} \\
\varepsilon_{1} & =e_{1}-e_{2}-e_{3}+e_{4} \\
\varepsilon_{2} & =e_{1}-e_{2}+e_{3}-e_{4} \\
\varepsilon_{3} & =e_{1}+e_{2}-e_{3}-e_{4}=\varepsilon_{1} \varepsilon_{2}
\end{aligned}
$$

This basis is just obtained by using the Fourier transform of the group $\mathbb{Z}_{2} \times \mathbb{Z}_{2}$. We have $\varepsilon_{i}^{2}=1, \varepsilon_{i} \varepsilon_{j}=\varepsilon_{j} \varepsilon_{i}=\varepsilon_{\langle i, j\rangle}$ for $i \neq j, \varepsilon_{i}^{*}=\varepsilon_{i}$, and these relations define a presentation of $\mathbb{C}^{4}$. Consider the linear map $\alpha: \mathbb{C}^{4} \rightarrow \mathbb{C}^{4} \otimes \mathrm{C}\left(S O_{-1}(3)\right)$ defined by $\alpha(1)=1 \otimes 1$ and $\alpha\left(\varepsilon_{i}\right)=\sum_{j} \varepsilon_{j} \otimes a_{j i}$. It is clear that $\alpha$ is coassociative, and it remains to check that $\alpha$ is a $*$-algebra morphism. We have

$$
\begin{aligned}
\alpha\left(\varepsilon_{i}\right)^{2} & =\sum_{k, l} \varepsilon_{k} \varepsilon_{l} \otimes a_{k i} a_{l i} \\
& =\sum_{k} \varepsilon_{k}^{2} \otimes a_{k i}^{2}+\sum_{k \neq l} \varepsilon_{k} \varepsilon_{l} \otimes a_{k i} a_{l i} \\
& =1 \otimes\left(\sum_{k} a_{k i}^{2}\right)+\sum_{k<l} \varepsilon_{k} \varepsilon_{l} \otimes\left(a_{k i} a_{l i}+a_{l i} a_{k i}\right) \\
& =1 \otimes 1=\alpha\left(\varepsilon_{i}^{2}\right)
\end{aligned}
$$

By using Lemma 3.1, we have, for $i \neq j$,

$$
\begin{aligned}
\alpha\left(\varepsilon_{i}\right) \alpha\left(\varepsilon_{j}\right) & =\sum_{k, l} \varepsilon_{k} \varepsilon_{l} \otimes a_{k i} a_{l j} \\
& =\sum_{k} \varepsilon_{k}^{2} \otimes a_{k i} a_{k j}+\sum_{k \neq l} \varepsilon_{k} \varepsilon_{l} \otimes a_{k i} a_{l j} \\
& =1 \otimes\left(\sum_{k} a_{k i} a_{k j}\right)+\sum_{k<l} \varepsilon_{k} \varepsilon_{l} \otimes\left(a_{k i} a_{l j}+a_{l i} a_{k j}\right) \\
& =\sum_{k<l} \varepsilon_{\langle k, l\rangle} \otimes a_{\langle k, l\rangle\langle i, j\rangle} \\
& =\sum_{k} \varepsilon_{k} \otimes a_{k\langle i, j\rangle}=\alpha\left(\varepsilon_{\langle i, j\rangle}\right)
\end{aligned}
$$

and since $\alpha\left(\varepsilon_{i}^{*}\right)=\alpha\left(\varepsilon_{i}\right)$, we conclude that $\alpha$ is a coaction.

Consider now a Woronowicz algebra $A$ coacting on $\mathbb{C}^{4}$, with coaction $\beta: \mathbb{C}^{4} \rightarrow$ $\mathbb{C}^{4} \otimes A$. We put $\varepsilon_{0}=1$ and we can write

$$
\beta\left(\varepsilon_{i}\right)=\sum_{j} \varepsilon_{j} \otimes x_{j i}
$$

with $x_{j 0}=\delta_{j 0}$. We have $x_{i j}^{*}=x_{i j}$ since $\beta$ is a $*$-morphism. Let $\phi: \mathbb{C}^{4} \rightarrow \mathbb{C}$ be the classical normalized integration map: $\phi\left(e_{i}\right)=1 / 4$, and hence $\phi\left(\varepsilon_{i}\right)=0$ if $i>0$. The linear map $\phi$ is $A$-colinear, and thus the linear map $\mathbb{C}^{4} \otimes \mathbb{C}^{4} \rightarrow \mathbb{C}, x \otimes y \mapsto \phi(x y)$, is $A$-colinear. It follows that the matrix $x=\left(x_{i j}\right)$ is orthogonal, and hence

$$
x_{01}^{2}+x_{02}^{2}+x_{03}^{2}=0=x_{01} x_{01}^{*}+x_{02} x_{02}^{*}+x_{03} x_{03}^{*}
$$


Therefore $x_{0 i}=\delta_{0 i}$, the matrix $x^{\prime}=\left(x_{i j}\right)_{1 \leq i, j \leq 3}$ is orthogonal and

$$
\beta\left(\varepsilon_{i}\right)=\sum_{j=1}^{3} \varepsilon_{j} \otimes x_{j i}, 1 \leq i \leq 3 .
$$

We have

$$
\begin{aligned}
1 \otimes 1 & =\beta(1)=\beta\left(\varepsilon_{i}^{2}\right)=\beta\left(\varepsilon_{i}\right)^{2} \\
& =\sum_{k, l} \varepsilon_{k} \varepsilon_{l} \otimes x_{k i} x_{l i} \\
& =\sum_{k} \varepsilon_{k}^{2} \otimes x_{k i}^{2}+\sum_{k \neq l} \varepsilon_{k} \varepsilon_{l} \otimes x_{k i} x_{l i} \\
& =1 \otimes\left(\sum_{k} x_{k i}^{2}\right)+\sum_{k<l} \varepsilon_{k} \varepsilon_{l} \otimes\left(x_{k i} x_{l i}+x_{l i} x_{k i}\right) \\
& =1 \otimes 1+\sum_{k<l} \varepsilon_{k} \varepsilon_{l} \otimes\left(x_{k i} x_{l i}+x_{l i} x_{k i}\right)
\end{aligned}
$$

and hence for $k \neq l$, we have $x_{k i} x_{l i}=-x_{l i} x_{k i}$, and using the antipode $\left(S\left(x_{i j}\right)=x_{j i}\right.$ since the matrix $x^{\prime}$ is orthogonal), we also get $x_{i k} x_{i l}=-x_{i l} x_{i k}$. For $i \neq j$, we have

$$
\begin{aligned}
\alpha\left(\varepsilon_{\langle i, j\rangle}\right) & =\alpha\left(\varepsilon_{i} \varepsilon_{j}\right)=\alpha\left(\varepsilon_{i}\right) \alpha\left(\varepsilon_{j}\right)=\sum_{k, l} \varepsilon_{k} \varepsilon_{l} \otimes x_{k i} x_{l j} \\
& =\sum_{k} \varepsilon_{k}^{2} \otimes x_{k i} x_{k j}+\sum_{k \neq l} \varepsilon_{k} \varepsilon_{l} \otimes x_{k i} x_{l j} \\
& =1 \otimes\left(\sum_{k} x_{k i} x_{k j}\right)+\sum_{k<l} \varepsilon_{k} \varepsilon_{l} \otimes\left(x_{k i} x_{l j}+x_{l i} x_{k j}\right) \\
& =\sum_{k<l} \varepsilon_{\langle k, l\rangle} \otimes\left(x_{k i} x_{l j}+x_{l i} x_{k j}\right)
\end{aligned}
$$

and hence $x_{\langle k, l\rangle\langle i, j\rangle}=x_{k i} x_{l j}+x_{l i} x_{k j}$. Similarly, since $\varepsilon_{i} \varepsilon_{j}=\varepsilon_{j} \varepsilon_{i}$, we have $x_{\langle k, l\rangle\langle i, j\rangle}=$ $x_{k j} x_{l i}+x_{l j} x_{k i}$. By combining these two relations, we get, for $i \neq j$ and $k \neq l$,

$$
\left[x_{k i}, x_{l j}\right]=\left[x_{k j}, x_{l i}\right]
$$

Now using the antipode, we also have

$$
\left[x_{k i}, x_{l j}\right]=\left[x_{l i}, x_{k j}\right]
$$

This finally leads to

$$
\left[x_{k i}, x_{l j}\right]=\left[x_{l i}, x_{k j}\right]=\left[x_{k j}, x_{l i}\right]=-\left[x_{l i}, x_{k j}\right]
$$

and we conclude that $x_{l i} x_{k j}=x_{k j} x_{l i}$. The quantum determinant relation now follows.

$$
\begin{aligned}
& x_{11} x_{22} x_{33}+x_{11} x_{23} x_{32}+x_{12} x_{21} x_{33}+x_{12} x_{23} x_{31}+x_{13} x_{22} x_{31}+x_{13} x_{23} x_{31} \\
= & x_{11}\left(x_{22} x_{33}+x_{23} x_{32}\right)+x_{12}\left(x_{21} x_{33}+x_{23} x_{31}\right)+x_{13}\left(x_{22} x_{31}+x_{23} x_{31}\right) \\
= & x_{11}^{2}+x_{12}^{2}+x_{13}^{2}=1
\end{aligned}
$$


Therefore we get a Woronowicz algebra morphism $\mathrm{C}\left(\mathrm{SO}_{-1}(3)\right) \rightarrow A$ commuting with the respective coactions, and we are done.

Remark. The proof of the theorem furnishes the following concrete isomorphism $\mathrm{C}\left(S O_{-1}(3)\right) \simeq \mathrm{C}\left(\mathcal{Q}_{4}\right):$

$$
\left(\begin{array}{cccc}
1 & 0 & 0 & 0 \\
0 & a_{11} & a_{12} & a_{13} \\
0 & a_{21} & a_{22} & a_{23} \\
0 & a_{31} & a_{32} & a_{33}
\end{array}\right) \longmapsto \frac{1}{4} M\left(\begin{array}{cccc}
u_{11} & u_{12} & u_{13} & u_{14} \\
u_{21} & u_{22} & u_{23} & u_{24} \\
u_{31} & u_{32} & u_{33} & u_{34} \\
u_{41} & u_{42} & u_{43} & u_{44}
\end{array}\right) M
$$

where

$$
M=\left(\begin{array}{cccc}
1 & 1 & 1 & 1 \\
1 & -1 & -1 & 1 \\
1 & -1 & 1 & -1 \\
1 & 1 & -1 & -1
\end{array}\right)
$$

The very first consequence of the isomorphism theorem is the following result.

Proposition 3.1. Any irreducible Hilbert space representation of the $\mathrm{C}^{*}$-algebra $A_{s}(4)=\mathrm{C}\left(\mathcal{Q}_{4}\right)$ is finite dimensional, and has dimension 1,2 or 4 .

Proof. We just have to prove the result for $\mathrm{C}\left(\mathrm{SO}_{-1}(3)\right)$. Let

$$
\pi: \mathrm{C}\left(S O_{-1}(3)\right) \rightarrow \mathcal{B}(H)
$$

be an irreducible representation on a Hilbert space $H$. For simplicity we just write $A=\mathrm{C}\left(S O_{-1}(3)\right)$. The elements $a_{i j}^{2}$ belong to the center of $A$, hence $\pi\left(a_{i j}^{2}\right)$ is a scalar. Using this fact and the commutation and anticommutation relations in $A$, we see that $\pi(A)$ is finite dimensional with $\operatorname{dim}(\pi(A)) \leq 2^{9}$. Il follows that $H$ is finite-dimensional. The relations of Lemma 3.1

$$
\begin{aligned}
& a_{13}=a_{21} a_{32}+a_{22} a_{31} \\
& a_{23}=a_{11} a_{32}+a_{12} a_{31} \\
& a_{33}=a_{11} a_{22}+a_{12} a_{21}
\end{aligned}
$$

now ensure that $\operatorname{dim}(\pi(A)) \leq 2^{6}$. On each column of the orthogonal matrix $\left(\pi\left(a_{i j}\right)\right)$, there is at least a nonzero element, which is invertible by the irreducibility of $\pi$, and from this we see that $\operatorname{dim}(\pi(A)) \leq 2^{5}$. If $n=\operatorname{dim}(H)$, we have $n^{2}=\operatorname{dim}(\pi(A))$, and hence $n \leq \sqrt{2} \cdot 4<6$. We also see from the anticommutation relations that if $n \neq 1$, then $n$ is necessarily even: this completes the proof.

\section{TWistings}

We discuss here the 2-cocycle deformation procedure, that we call twisting, at a purely algebraic level. This method was initiated by Drinfeld, and studied in a systematic way in the dual framework by Doi [18].

Let $H$ be a Hopf algebra. We use Sweedler's notation $\Delta(x)=x_{1} \otimes x_{2}$. Recall (see e.g. [18]) that a 2-cocycle is a convolution invertible linear map $\sigma: H \otimes H \longrightarrow \mathrm{C}$ satisfying

$$
\sigma\left(x_{1}, x_{2}\right) \sigma\left(x_{2} y_{2}, z\right)=\sigma\left(y_{1}, z_{1}\right) \sigma\left(x, y_{2} z_{2}\right)
$$

and $\sigma(x, 1)=\sigma(1, x)=\varepsilon(x)$, for $x, y, z \in H$. 
Following [18] and [36], we associate various algebras to a 2-cocycle. First consider the algebra ${ }_{\sigma} H$. As a vector space we have ${ }_{\sigma} H=H$ and the product of ${ }_{\sigma} H$ is defined to be

$$
\{x\}\{y\}=\sigma\left(x_{1}, y_{1}\right)\left\{x_{2} y_{2}\right\}, \quad x, y \in H,
$$

where an element $x \in H$ is denoted $\{x\}$, when viewed as an element of ${ }_{\sigma} H$.

We also have the algebra $H_{\sigma^{-1}}$, where $\sigma^{-1}$ denotes the convolution inverse of $\sigma$. As a vector space we have $H_{\sigma^{-1}}=H$ and the product of $H_{\sigma^{-1}}$ is defined to be

$$
\langle x\rangle\langle y\rangle=\sigma^{-1}\left(x_{2}, y_{2}\right)\left\langle x_{1} y_{1}\right\rangle, \quad x, y \in H .
$$

where an element $x \in H$ is denoted $\langle x\rangle$, when viewed as an element of $H_{\sigma^{-1}}$. The cocycle condition ensures that ${ }_{\sigma} H$ and $H_{\sigma^{-1}}$ are associative algebras with 1 as a unit.

Finally we have the Hopf algebra $H^{\sigma}={ }_{\sigma} H_{\sigma^{-1}}$. As a coalgebra $H^{\sigma}=H$. The product of $H^{\sigma}$ is defined to be

$$
[x][y]=\sigma\left(x_{1}, y_{1}\right) \sigma^{-1}\left(x_{3}, y_{3}\right)\left[x_{2} y_{2}\right], \quad x, y \in H,
$$

where an element $x \in H$ is denoted $[x]$, when viewed as an element of $H^{\sigma}$, and we have the following formula for the antipode of $H^{\sigma}$ :

$$
S^{\sigma}([x])=\sigma\left(x_{1}, S\left(x_{2}\right)\right) \sigma^{-1}\left(S\left(x_{4}\right), x_{5}\right)\left[S\left(x_{3}\right)\right] .
$$

The Hopf algebras $H$ and $H^{\sigma}$ have equivalent tensor categories of comodules [36].

Very often 2-cocyles are induced by simpler quotient Hopf algebras (quantum subgroups). More precisely let $\pi: H \rightarrow K$ be a Hopf algebra surjection and let $\sigma: K \otimes K \rightarrow \mathbb{C}$ be a 2-cocycle on $K$. Then $\sigma_{\pi}=\sigma \circ(\pi \otimes \pi): H \otimes H \rightarrow \mathbb{C}$ is a 2-cocycle.

Lemma 4.1. Let $\pi: H \rightarrow K$ be a Hopf algebra surjection and let $\sigma: K \otimes K \rightarrow \mathbb{C}$ be a 2-cocycle on $K$. We have an injective algebra map

$$
\begin{aligned}
\theta: H^{\sigma_{\pi}} & \longrightarrow{ }_{\sigma} K \otimes H \otimes K_{\sigma^{-1}} \\
{[x] } & \longmapsto\left\{\pi\left(x_{1}\right)\right\} \otimes x_{2} \otimes\left\langle\pi\left(x_{3}\right)\right\rangle
\end{aligned}
$$

Proof. It is a direct verification that $\theta$ is an algebra map, and the injectivity follows by using the counit of $K$, available as a linear map.

This lemma, when $K$ is finite dimensional and $H$ is commutative, furnishes a faithful representation of $H^{\sigma_{\pi}}$.

The following lemma will be used in the section concerning the classification.

Lemma 4.2. Let $\pi_{1}, \pi_{2}: H \rightarrow K$ be two Hopf algebra surjections and let $\sigma: K \otimes$ $K \rightarrow \mathbb{C}$ be a 2-cocycle on $K$. Assume that there exists a Hopf algebra automorphism $u: H \rightarrow H$ such that $\pi_{2} \circ u=\pi_{1}$. Then the Hopf algebra $H^{\sigma_{\pi_{1}}}$ and $H^{\sigma_{\pi_{2}}}$ are isomorphic.

Proof. The announced isomorphism is the map $H^{\sigma_{\pi_{1}}} \rightarrow H^{\sigma_{\pi_{2}}},[x] \mapsto[u(x)]$.

The following lemma is useful to classify certain quotient Hopf algebras.

Lemma 4.3. Let $\pi: H \rightarrow K$ be a Hopf algebra surjection and let $\sigma: K \otimes K \rightarrow \mathbb{C}$ be a 2-cocycle. Then there is a bijection between the following data. 
(1) Surjective Hopf algebra maps $f: H \rightarrow L$ such that there exists a Hopf algebra map $g: L \rightarrow K$ satisfying $g \circ f=\pi$.

(2) Surjective Hopf algebra maps $f^{\prime}: H^{\sigma_{\pi}} \rightarrow L^{\prime}$ such that there exists a Hopf algebra map $g^{\prime}: L^{\prime} \rightarrow K^{\sigma}$ satisfying $g^{\prime} \circ f^{\prime}=[\pi]$.

Assume moreover that $H$ and $K$ are Hopf $*$-algebras, that $\pi$ is a Hopf $*$-algebra map and that $H^{\sigma_{\pi}}$ and $K^{\sigma}$ admit Hopf *-algebra structures induced by those of $H$ and $K$ respectively (i.e. $[x]^{*}=\left[x^{*}\right]$ ). Then the above correspondence remains true for Hopf *-algebras and Hopf *-algebra surjections.

Proof. Let us start with surjective Hopf algebra maps $f: H \rightarrow L$ and $g: L \rightarrow K$ satisfying $g \circ f=\pi$. Then the Hopf algebra maps $[f]: H^{\sigma_{\pi}} \rightarrow L^{\sigma_{g}}$ and $[g]: L^{\sigma_{g}} \rightarrow$ $K^{\sigma}$ satisfy $[g] \circ[f]=[\pi]$. We get the other side of the correspondence by using the cocycle $\sigma^{-1}$. The Hopf $*$-algebra assertion is then immediate.

We conclude the section by mentioning the pseudo-twisting procedure, considered in the dual framework in $[20,38,33]$. Consider a convolution invertible map $\sigma$ : $H \otimes H \rightarrow \mathbb{C}$. If $\sigma$ is a pseudo-2-cocycle, it is possible to define a Hopf algebra $H^{\sigma}$ as above, and $H^{\sigma}$ has the same fusion semiring as $H$. Some examples of pseudo-twisted Hopf algebras will occur in Section 7.

\section{Twisting And QUantum $S O_{-1}(3)$}

We now proceed to show that the quantum group $S O_{-1}(3)$ of the previous section may be obtained by twisting the group $S O(3)$.

Let $H$ be the algebra of representative functions on the compact group $S O(3)$, with the canonical coordinate functions denoted $x_{i j}$. We consider the Klein group, denoted $\mathrm{V}$ as usual,

$$
\mathrm{V}=\mathbb{Z}_{2} \times \mathbb{Z}_{2}=\left\langle t_{1}, t_{2} \mid t_{1}^{2}=t_{2}^{2}=1, t_{1} t_{2}=t_{2} t_{1}\right\rangle
$$

and we put $t_{3}=t_{1} t_{2}$. The restriction of functions on $S O(3)$ to its diagonal subgroup gives a Hopf algebra surjection

$$
\begin{aligned}
\pi_{d}: H & \longrightarrow \mathrm{C}[\mathrm{V}] \\
x_{i j} & \longmapsto \delta_{i j} t_{i}
\end{aligned}
$$

Let $\sigma: \mathrm{V} \times \mathrm{V} \longrightarrow \mathbb{C}^{*}$ be the unique bimultiplicative map such that $\sigma\left(t_{i}, t_{j}\right)=-1$ if $i \leq j$ and $\sigma\left(t_{i}, t_{j}\right)=1$ otherwise. Then $\sigma$ is a usual group 2-cocycle, and its unique linear extension to the group algebra $\mathbb{C}[\mathrm{V}] \otimes \mathbb{C}[\mathrm{V}]$ is a 2-cocycle in the previous sense, still denoted $\sigma$. We get a cocycle $\sigma_{d}=\sigma \circ\left(\pi_{d} \otimes \pi_{d}\right)$ on $H$. Note that $\sigma_{d}=\sigma_{d}^{-1}$.

Theorem 5.1. The Hopf algebra $H^{\sigma_{d}}$ is a CQG-algebra, and the Woronowicz algebras $\mathrm{C}^{*}\left(H^{\sigma_{d}}\right)$ and $\mathrm{C}\left(S_{-1}(3)\right)$ are isomorphic.

Proof. We have

$$
\begin{aligned}
{\left[x_{i j}\right]\left[x_{k l}\right] } & =\sum_{p, q, r, s} \sigma_{d}\left(x_{i p}, x_{k q}\right) \sigma_{d}^{-1}\left(x_{r j}, x_{s l}\right)\left[x_{p r} x_{q s}\right] \\
& =\sigma\left(t_{i}, t_{k}\right) \sigma\left(t_{j}, t_{l}\right)\left[x_{i j} x_{k l}\right]
\end{aligned}
$$


and more generally

$$
\left[x_{i_{1} j_{1}}\right]\left[x_{i_{2} j_{2}}\right] \ldots\left[x_{i_{r} j_{r}}\right]=\left(\prod_{p<q} \sigma\left(t_{i_{p}}, t_{i_{q}}\right)\right)\left(\prod_{p<q} \sigma\left(t_{j_{p}}, t_{j_{q}}\right)\right)\left[x_{i_{1} j_{1}} x_{i_{2} j_{2}} \ldots x_{i_{r} j_{r}}\right]
$$

Thus we see that there is a Hopf algebra morphism $\mathcal{R}\left(S O_{-1}(3)\right) \rightarrow H^{\sigma_{d}}$ mapping $a_{i j}$ to $\left[x_{i j}\right]$. This morphism is clearly surjective. Now combining Theorem 3.1 and [2], we see that the representation semiring of $\mathcal{R}\left(S O_{-1}(3)\right)$ is the same as the one of $S O(3)$, and thus by a standard argument the two cosemisimple Hopf algebras $\mathcal{R}\left(S O_{-1}(3)\right)$ and $H^{\sigma_{d}}$ are isomorphic. The theorem follows.

We now recover the faithful representation of [11], under a different form. The same kind of embedding was constructed for $S U_{-1}(2)$ in [45]. It will be used in the next section. First we need the following lemma, whose proof is immediate.

Lemma 5.1. Let $H$ be a cosemisimple Hopf algebra with Haar measure $h: H \rightarrow \mathbb{C}$, and let $\pi: H \rightarrow K$ be a Hopf algebra morphism. Let $\varphi: K \rightarrow \mathbb{C}$ be a linear map such that $\varphi(1)=1$. Then for $x \in H$, we have

$$
h(x)=\varphi\left(\pi\left(x_{1}\right)\right) \varphi\left(\pi\left(x_{3}\right)\right) h\left(x_{2}\right)
$$

We use the Pauli matrices

$$
\tau_{1}=\left(\begin{array}{ll}
i & 0 \\
0 & -i
\end{array}\right), \quad \tau_{2}=\left(\begin{array}{ll}
0 & 1 \\
-1 & 0
\end{array}\right), \quad \tau_{3}=\tau_{2} \tau_{1}=\left(\begin{array}{ll}
0 & -i \\
-i & 0
\end{array}\right)
$$

Theorem 5.2. We have a $\mathrm{C}^{*}$-algebra embedding

$$
\begin{aligned}
& \theta: \mathrm{C}\left(S O_{-1}(3)\right) \longrightarrow M_{2}(\mathbb{C}) \otimes M_{2}(\mathbb{C}) \otimes \mathrm{C}(S O(3)) \\
& a_{i j} \longmapsto \tau_{i} \otimes \tau_{j} \otimes x_{i j}
\end{aligned}
$$

Proof. The first thing to note is that the twisted group algebra $\mathbb{C}[\mathrm{V}]_{\sigma}=\mathbb{C}_{\sigma}[\mathrm{V}]$ is isomorphic to $M_{2}(\mathbb{C})$ via $\left\{t_{i}\right\} \mapsto \tau_{i}$. Then combining this with Theorem 5.1 and Lemma 4.1 , we get a $*$-algebra embedding $f: \mathcal{R}\left(S O_{-1}(3)\right) \rightarrow M_{2}(\mathbb{C}) \otimes M_{2}(\mathbb{C}) \otimes H$, inducing the announced $*$-algebra map. Let $\operatorname{tr}$ be the normalized trace on $M_{2}(\mathbb{C})$, and let $h$ be the Haar mesure on $H$. Then $(\operatorname{tr} \otimes \operatorname{tr} \otimes h) \circ f$ is, by the invariance of the Haar measure by cocycle twisting and Lemma 5.1, the Haar measure on $\mathcal{R}\left(S O_{-1}(3)\right)$. We can conclude as in [11], using the amenability of $\widehat{\mathcal{Q}_{4}}$, that the *-algebra morphism $\mathrm{C}\left(S O_{-1}(3)\right) \longrightarrow M_{2}(\mathbb{C}) \otimes M_{2}(\mathbb{C}) \otimes \mathrm{C}(S O(3))$ is injective.

\section{Quantum subgroups of $\mathcal{Q}_{4}$}

We now study the quantum subgroups of $\mathcal{Q}_{4}$. They correspond to Woronowicz algebra quotients of $\mathrm{C}\left(\mathcal{Q}_{4}\right)$ and hence of $\mathrm{C}\left(S O_{-1}(3)\right)$, or equivalently to Hopf $*$ algebra quotients of $\mathcal{R}\left(S_{-1}(3)\right)$. We work at the Hopf algebra level. The strategy for proving Theorem 1.1 is the following one. Our first goal is the classification of the quantum subgroups of $S_{-1}(3)$ diagonally containing the Klein subgroup (see the notation below). This is achieved in Theorem 6.1, by using the technical results 6.1-6.5. Then another technical result, Lemma 6.6, shows that the general classification follows from the classification follows from the classification for some proper quantum subgroups. The classification is then performed for these quantum 
subgroups, with the exception of the quantum group $O_{-1}(2)$, that will be treated in the next section.

First we need some notations. Most of them have been introduced in the previous section.

(1) The Hopf $*$-algebra $H$ is the Hopf algebra $\mathcal{R}(S O(3))$, with the coordinate functions denoted $x_{i j}$. We denote by $I_{d}$ the ideal of $H$ generated by the elements $x_{i j}, i \neq j$.

(2) The Klein group is the group $\mathrm{V}=\mathbb{Z}_{2} \times \mathbb{Z}_{2}=\left\langle t_{1}, t_{2} \mid t_{1}^{2}=t_{2}^{2}=1, t_{1} t_{2}=t_{2} t_{1}\right\rangle$, and we put $t_{3}=t_{1} t_{2}$.

(3) The group of diagonal matrices in $S O(3)$ is denoted $D$. It is isomorphic with $\mathrm{V}$ via the injective group morphism

$$
t_{1} \longmapsto\left(\begin{array}{lll}
1 & 0 & 0 \\
0 & -1 & 0 \\
0 & 0 & -1
\end{array}\right), \quad t_{2} \longmapsto\left(\begin{array}{lll}
-1 & 0 & 0 \\
0 & 1 & 0 \\
0 & 0 & -1
\end{array}\right)
$$

(4) We have a "diagonal" Hopf $*$-algebra surjection $\pi_{d}: H \rightarrow \mathbb{C}[\mathrm{V}], x_{i j} \mapsto \delta_{i j} t_{i}$, induced by the previous diagonal embedding $\mathrm{V} \hookrightarrow S O(3)$. It is clear that $I_{d}=\operatorname{ker}\left(\pi_{d}\right)$.

(5) The 2-cocycle on $\mathrm{V}$ defined in the previous section is denoted $\sigma$, and it induces a 2-cocycle $\sigma_{d}$ on $H$. More generally, if $K$ is a Hopf algebra and $f: K \rightarrow \mathbb{C}[\mathrm{V}]$ is a Hopf algebra map, then $\sigma_{f}=\sigma \circ(f \otimes f)$ is a 2-cocycle on $K$.

(6) The Hopf $*$-algebra $H^{\prime}$ is the Hopf algebra $\mathcal{R}\left(S O_{-1}(3)\right)$. Thanks to Theorem 5.1 in the previous section, we identify it with $H^{\sigma_{d}}$. We have a diagonal surjective Hopf $*$-algebra map $\pi_{d}^{\prime}: H^{\prime} \rightarrow \mathbb{C}[\mathrm{V}], a_{i j} \mapsto \delta_{i j} t_{i} . \pi_{d}^{\prime}$ induces a quantum group embedding $\mathrm{V} \subset S O_{-1}(3)$, called the diagonal embedding of $\mathrm{V}$ into $S O_{-1}(3)$. In fact $\pi_{d}^{\prime}$ is just $\left[\pi_{d}\right]$, after the identification $H^{\prime}=H^{\sigma_{d}}$. We put $I_{d}^{\prime}=\operatorname{ker}\left(\pi_{d}^{\prime}\right)$. Clearly this is the ideal generated by the elements $a_{i j}$, $i \neq j$.

6.1. Quantum subgroups diagonally containing the Klein subgroup. We begin with a lemma.

Lemma 6.1. Let $X \subset S O(3)$ be a compact subgroup with $D \subset X$, and let $g$ : $\mathcal{R}(X) \rightarrow \mathbb{C}[\mathrm{V}]$ be the corresponding Hopf *-algebra surjection. Then $\mathcal{R}(X)^{\sigma_{g}}$ is a quotient Hopf *-algebra of $\mathcal{R}\left(S O_{-1}(3)\right)$.

Conversely, let $G \subset S O_{-1}(3)$ be a compact quantum subgroup, and let $f: H^{\prime} \rightarrow$ $\mathcal{R}(G)$ be the corresponding surjective Hopf *-algebra map. Assume that there exists a Hopf *-algebra map $g^{\prime}: \mathcal{R}(G) \rightarrow \mathbb{C}[\mathrm{V}]$ such that $g^{\prime} \circ f=\pi_{d}^{\prime}$ : the corresponding composition of embeddings $\mathrm{V} \subset G \subset S O_{-1}(3)$ is the diagonal embedding. Then there exists a compact subgroup $X$ of $S O(3)$ with $D \subset X \subset S O(3)$, with a Hopf *-algebra isomorphism

$$
\mathcal{R}(G) \simeq \mathcal{R}(X)^{\omega}
$$

for a 2-cocyle $\omega$ induced by $\sigma$.

Proof. The Hopf *-algebra quotients of $H=\mathcal{R}(S O(3))$ all are isomorphic with $\mathcal{R}(X)$, for a compact subgroup $X \subset S O(3)$. Hence this lemma is just a reformulation of Lemma 4.3 annd its proof. 
Using the well-known list of compact subgroups of $S O(3)$, we will get the first classification result. The subgroups containing $D$ are: $S O(3), O(2), S_{4}, A_{4}, D_{4}$, $D \simeq \mathbb{Z}_{2} \times \mathbb{Z}_{2}, D_{n}$ with $n>4$ even and $A_{5}$.

We need the following lemma, which collects various results on 2-cocycles deformations in the litterature.

Lemma 6.2. The groups $A_{4}, D_{4}$ and $\mathbb{Z}_{2} \times \mathbb{Z}_{2}$ do not have any non-trivial twist, and the groups $S_{4}, A_{5}$ and $D_{n}$ for $n>4$ even, have exactly one non-trivial twist.

Proof. The assertion for the dihedral groups is due to Masuoka [30], and for $\mathbb{Z}_{2} \times \mathbb{Z}_{2}$ this is trivial. For the other groups we have the following general fact, due to Etingof-Gelaki [21, 23] and Davydov [16]. If $G$ is a finite group, any non-trivial twist of $\mathrm{C}(G)$ arises from the following data: a non-normal subgroup $K$ endowed with a (necessarly non trivial) 2-cocycle $\alpha$ on $K$ such that the twisted group algebra of $\mathbb{C}_{\alpha}[K]$ is a matrix algebra. For the groups $A_{4}, S_{4}$ and $A_{5}$, the only possible candidate for $K$ is a Klein subgroup $\mathrm{V}$, which is normal (and hence unique) for $A_{4}$, and hence we have the assertion for $A_{4}$. Also there is only one non trivial cohomology class in $H^{2}\left(\mathrm{~V}, \mathbb{C}^{*}\right)$, and since the non-normal Klein subgroups of these groups are all conjugate, by Lemma 4.2 there is at most one possible twist for $S_{4}$ and $A_{5}$. These twists were constructed by Nikshych [33].

Lemma 6.3. The compact groups $S O(3), O(2), D_{n}$ with $n$ even and $n>4$, and $A_{5}$ are not quantum subgroups of $\mathrm{SO}_{-1}(3)$.

Proof. This follows from the fact that $\mathrm{C}\left(S_{4}\right)$ is the maximal abelian quotient of $\mathcal{R}\left(\mathcal{Q}_{4}\right) \simeq \mathcal{R}\left(S O_{-1}(3)\right)$.

Lemma 6.4. The finite quantum groups $D_{n}^{\tau}, n$ even and $n>4, S_{4}^{\tau}$, and $A_{5}^{\tau}$, the respective unique non-trivial twists of $D_{n}, S_{4}$ and $A_{5}$, all occur as quantum subgroups of $\mathrm{SO}_{-1}(3)$ diagonally containing $\mathrm{V}$.

Proof. We know from Lemma 6.1 that for a compact group $X$ with $D \subset X \subset S O(3)$, there exists a 2-cocycle $\omega$ on $\mathcal{R}(X)$ such that $\mathcal{R}(X)^{\omega}$ is a quotient Hopf $*$-algebra of $\mathcal{R}\left(S O_{-1}(3)\right)$. Using the previous lemma, we see that $D_{n}^{\tau}$ anf $A_{5}^{\tau}$ are quantum subgroups of $S O_{-1}(3)$ diagonally containing $\mathrm{V}$. The quantum group $S_{4}^{\tau}$ is realized as a quantum subgroup of $\mathcal{Q}_{4}$ in [13]. Still denoting by $u_{i j}$ the generators of $\mathrm{C}\left(S_{4}^{\tau}\right)$, we have, by using the presentation of $\mathrm{C}\left(S_{4}^{\tau}\right)$ given in [13], a surjective Hopf *-algebra $\operatorname{map} \mathrm{C}\left(S_{4}^{\tau}\right) \rightarrow \mathbb{C}[\mathrm{V}]$

$$
\left(\begin{array}{llll}
u_{11} & u_{12} & u_{13} & u_{14} \\
u_{21} & u_{22} & u_{23} & u_{24} \\
u_{31} & u_{32} & u_{33} & u_{34} \\
u_{41} & u_{42} & u_{43} & u_{44}
\end{array}\right) \longmapsto\left(\begin{array}{llll}
\delta_{1} & \delta_{t_{1}} & \delta_{t_{2}} & \delta_{t_{3}} \\
\delta_{t_{1}} & \delta_{1} & \delta_{t_{3}} & \delta_{t_{2}} \\
\delta_{t_{2}} & \delta_{t_{3}} & \delta_{1} & \delta_{t_{1}} \\
\delta_{t_{3}} & \delta_{t_{2}} & \delta_{t_{1}} & \delta_{1}
\end{array}\right)
$$

Composed with the canonical morphisms $\mathcal{R}\left(S O_{-1}(3)\right) \simeq \mathcal{R}\left(\mathcal{Q}_{4}\right) \rightarrow C\left(S_{4}^{\tau}\right)$, this gives embeddings $V \subset S_{4}^{\tau} \subset S O_{-1}(3)$, and the composition is the diagonal embedding.

We will also need the quantum group $O_{-1}(2)$, also considered in [8].

Definition 6.1. The *-algebra $\left.\mathcal{R}\left(O_{-1}(2)\right)\right)$ is the $*$-algebra presented by generators $\left(a_{i j}\right)_{1 \leq i, j \leq 2}$ and submitted to the following relations. 
(1) The matrix $a=\left(a_{i j}\right)$ is orthogonal,

(2) $a_{i j} a_{i k}=-a_{i k} a_{i j}$ and $a_{j i} a_{j k}=-a_{j k} a_{j i}$, for $j \neq k$,

(3) $a_{i j} a_{k l}=a_{k l} a_{i j}$, for $i \neq k$ and $j \neq l$,

It is immediate to check that $\mathcal{R}\left(O_{-1}(2)\right)$ is a Hopf $*$-algebra with

$$
\Delta\left(a_{i j}\right)=\sum_{k} a_{i k} \otimes a_{k j}, \varepsilon\left(a_{i j}\right)=\delta_{i j}, S\left(a_{i j}\right)=a_{j i}
$$

and is a CQG-algebra. Also it is easy to construct a surjective Hopf *-algebra map $\mathcal{R}\left(S O_{-1}(3)\right) \rightarrow \mathcal{R}\left(O_{-1}(2)\right)$, yielding an embedding $O_{-1}(2) \subset S O_{-1}(3)$ such that the composition of the embeddings $\mathrm{V} \subset O_{-1}(2) \subset S O_{-1}(3)$ is the diagonal one.

Lemma 6.5. The Hopf *-algebra $\mathcal{R}\left(O_{-1}(2)\right)$ is a twist of $\mathcal{R}(O(2))$, by a 2-cocycle induced by the diagonal Klein subgroup of $O(2)$. Moreover this is the only non-trivial such twist of $\mathcal{R}(O(2))$.

Proof. The first assertion is proved in [8] where it is shown that $O_{-1}(2)$ is the quantum symmetry group of the square, see also [15]. All the Klein subgroups of $O(2)$ are conjugate, and we have the uniqueness result using Lemma 4.2.

Combining the above results, we can state the first classification result.

Theorem 6.1. The compact quantum subgroups $G$ of $S O_{-1}(3)$ with $\mathrm{V} \subset G$, and such that the composition of the embeddings $\mathrm{V} \subset G \subset S O_{-1}(3)$ is the diagonal embedding, are exactly, up to isomorphism, the following ones.

(1) $\mathrm{SO}_{-1}(3)$.

(2) $\mathrm{O}_{-1}(2)$.

(3) $\mathbb{Z}_{2} \times \mathbb{Z}_{2}, D_{4}, A_{4}$ or $S_{4}$.

(4) $D_{n}^{\tau}$, $n$ even and $n>4$, the only non-trivial twist of the dihedral group $D_{n}$.

(5) $S_{4}^{\tau}$, the only non-trivial twist of the symmetric group $S_{4}$.

(6) $A_{5}^{\tau}$, the only non-trivial twist of the alternating group $A_{5}$.

6.2. The general case. We now deal with general quantum subgroups. So let us consider a Hopf $*$-algebra surjection $f: H^{\prime}=\mathcal{R}\left(S O_{-1}(3)\right) \rightarrow L$ and let $J=\operatorname{ker} f$. Consider the Hopf $*$-algebra $\tilde{L}=L /\left(J \cap I_{d}^{\prime}\right)$. It is clear that $\tilde{L}=\mathcal{R}(G)$ for one of the quantum groups of Theorem 6.1. The following lemma ensures that if $\tilde{L}$ is $\mathcal{R}\left(S O_{-1}(3)\right)$, then already $L$ was $\mathcal{R}\left(S O_{-1}(3)\right)$.

Lemma 6.6. Let $J$ be a Hopf $*$-ideal in $H^{\prime}$. If $J \cap I_{d}^{\prime}=(0)$, then $J=(0)$.

Proof. Consider the embedding

$$
\begin{aligned}
& \theta: \mathrm{C}\left(S O_{-1}(3)\right) \longrightarrow M_{2}(\mathbb{C}) \otimes M_{2}(\mathbb{C}) \otimes \mathrm{C}(S O(3)) \\
& a_{i j} \longmapsto \tau_{i} \otimes \tau_{j} \otimes x_{i j}
\end{aligned}
$$

in Theorem 5.2. Let $\tau_{0}$ be the identity matrix. Then $\left(\tau_{i} \otimes \tau_{j}\right)_{0 \leq i, j \leq 3}$ is a basis of $M_{2}(\mathbb{C}) \otimes M_{2}(\mathbb{C})$. Hence for $x \in H^{\prime}$, there exists elements $p_{i j} \in H$ such that $\theta(x)=\sum_{i, j} \tau_{i} \otimes \tau_{j} \otimes p_{i j}$. Now assume that $x \in J$. For $k \neq l$, we have $a_{k l} x \in J \cap I_{d}^{\prime}$, hence $a_{k l} x=0$, and

$$
\theta\left(a_{k l} x\right)=\sum_{i, j} \tau_{k} \tau_{i} \otimes \tau_{l} \tau_{j} \otimes x_{k l} p_{i j}=0
$$


It follows that $x_{k l} p_{i j}=0$ for all $i$ and $j$. The algebra $H$ is an integral domain (the algebraic group $S O(3)$ is irreducible), hence $p_{i j}=0, \forall i, j$. Therefore $\theta(x)=0$ and $x=0$.

Therefore, to complete the classification, it remains to find the quantum subgroups of the following quantum groups.

(1) $O_{-1}(2)$.

(2) $D_{n}^{\tau}, n$ even and $n>4$.

(3) $S_{4}^{\tau}$, the only non-trivial twist of the symmetric group $S_{4}$.

(4) $A_{5}^{\tau}$, the only non-trivial twist of the alternating group $A_{5}$.

For the quantum orthogonal group $O_{-1}(2)$, this will be done in the next section. The twisted dihedral groups are quantum subgroups of $O_{-1}(2)$, and hence the classification of their quantum subgroups will follow from the one for $O_{-1}(2)$.

Thus it remains to examine the twists $S_{4}^{\tau}$ and $A_{5}^{\tau}$. We use in a crucial way some results of Etingof and Gelaki [22] to prove that their proper quantum subgroups are all classical groups.

Lemma 6.7. The proper quantum subgroups of $S_{4}^{\tau}$ are all classical subgroups of $D_{4}$.

Proof. We determine the algebra structure of the semisimple algebra $\mathrm{C}\left(S_{4}^{\tau}\right)$. First we know from [13] that the maximal abelian quotient of $\mathrm{C}\left(S_{4}^{\tau}\right)$ is $\mathrm{C}\left(D_{4}\right)$ and in particlar $C\left(S_{4}^{\tau}\right)$ has 8 non-equivalent one-dimensional representations. The 2-cocycle is induced by the Klein subgroup

$$
\mathrm{V}=\{(1,(1,2),(3,4),(1,2)(3,4)\}
$$

and is in minimal over $\mathrm{C}(\mathrm{V})$ in the sense of [22]. Let $g=(1,2,3)$. Following [22], we put $\mathrm{V}_{g}=\mathrm{V} \cap g \mathrm{Vg}^{-1}$. We have $\mathrm{V}_{g}=\{1\}$ and hence Theorem 3.2 in [22] furnishes an irreducible representation of $\mathrm{C}\left(S_{4}^{\tau}\right)$ having a dimension that is a multiple of 4 . An immediate counting argument (or Proposition 3.1) shows that this representation has dimension 4. Therefore we have an algebra isomorphism

$$
\mathrm{C}\left(S_{4}^{\tau}\right) \simeq \mathbb{C}^{8} \oplus M_{4}(\mathbb{C})
$$

Now if $L$ is a non-commutative Hopf algebra quotient of $\mathrm{C}\left(S_{4}^{\tau}\right)$, its dimension is at least 16 and divides 24 by the Nichols-Zoeller theorem [32]: we are done.

Lemma 6.8. The proper quantum subgroups of $A_{5}^{\tau}$ are all classical subgroups of $A_{4}$.

Proof. Again we begin by determining the algebra structure of $\mathrm{C}\left(A_{5}^{\tau}\right)$. Consider the Klein subgroup of $A_{5}$.

$$
\mathrm{V}=\{1,(1,2)(3,4),(1,3)(2,4),(1,4)(2,3)\}
$$

The group inclusions $V \subset A_{4} \subset A_{5}$ yield surjective Hopf algebra maps $\mathrm{C}\left(A_{5}\right) \rightarrow$ $\mathrm{C}\left(A_{4}\right) \rightarrow \mathrm{C}(\mathrm{V})$. Twisting by the unique non-trivial 2-cocycle on $V$ and using Lemma 6.2 and Lemma 6.4 now yields surjective Hopf algebra maps

$$
\mathrm{C}\left(A_{5}^{\tau}\right) \rightarrow \mathrm{C}\left(A_{4}\right) \rightarrow \mathrm{C}(\mathrm{V})
$$

This shows that $\mathrm{C}\left(A_{5}^{\tau}\right)$ has 12 non-equivalent one-dimensional representations and that the classical subgroups of $A_{5}^{\tau}$ are the subgroups of $A_{4}$. Let $g_{1}=(1,2,5)$ and put $\mathrm{V}_{g_{1}}=\mathrm{V} \cap g_{1} \mathrm{~V}_{1}^{-1}$. We have $V_{g_{1}}=\{1\}$ and hence Theorem 3.2 in [22] furnishes 
an irreducible representation $V_{1}$ of $\mathrm{C}\left(A_{5}^{\tau}\right)$ having a dimension that is a multiple of 4 , and since $\left(\operatorname{dim} V_{1}\right)^{2}<60$, we have $\operatorname{dim} V_{1}=4$. Now consider $g_{2}=(1,3,5)$ and $g_{3}=(1,4,5)$. The same reasonning as above furnishes irreducible 4-dimensional representations $V_{2}$ and $V_{3}$. For $i \neq j$ we have $\mathrm{V} g_{i} \mathrm{~V} \neq \mathrm{V} g_{j} \mathrm{~V}$ and by using [22], we see that the representations $V_{1}, V_{2}$ and $V_{3}$ are pairwise non-equivalent. We conclude that

$$
\mathrm{C}\left(A_{5}^{\tau}\right) \simeq \mathbb{C}^{12} \oplus M_{4}(\mathbb{C})^{3}
$$

Let $L$ be a non-commutative Hopf algebra quotient of $\mathrm{C}\left(A_{5}^{\tau}\right)$ : its dimension is at least 16 and divide 60 by the Nichols-Zoeller Theorem, thus $\operatorname{dim} L=20,30$ or 60 . A counting argument shows that $\operatorname{dim} L \neq 30$. Assume that $\operatorname{dim} L=20$. Then, as an algebra, $L \simeq \mathbb{C}^{4} \oplus M_{4}(\mathbb{C})$. Hence the maximal abelian quotient of $L$ is $\mathbb{C}^{4} \simeq \mathrm{C}(\mathrm{V})$, and we have Hopf algebra surjections $\mathrm{C}\left(A_{5}^{\tau}\right) \rightarrow L \rightarrow \mathrm{C}(\mathrm{V})$. Twisting by the appropriate 2-cocycle yields Hopf algebra surjections $\mathrm{C}\left(A_{5}\right) \rightarrow L^{\prime} \rightarrow \mathrm{C}(\mathrm{V})$. This means that $A_{5}$ has a subgroup of order 20, which is known to be false. Hence $\operatorname{dim} L=60$ and we are done.

\section{The QuAntum Group $O_{-1}(2)$ AND ITS QUANTUM SUbGroups}

In this section we classify the compact quantum subgroups of $O_{-1}(2)$, finishing in this way the proof of the classification theorem. The novelty will be the occurence of $\widehat{D}_{\infty}$, the quantum dual of the infinite dihedral group and of pseudo-twisted dicyclic groups $D C_{n}^{\tau}$.

First let us note that we can classify the quantum subgroups diagonally containing the Klein subgroup in a manner similar to the one of the previous section. In particular we conclude that the quantum groups $D_{n}^{\tau}, n>4$ and even, all are quantum subgroups of $O_{-1}(2)$. But the techniques of the end of the last section do not work here, because $O(2)$ is not connected. So we will proceed directly, and another presentation of $\mathcal{R}\left(O_{-1}(2)\right)$ will be more convenient.

Recall [8] that $A_{h}(2)$ is the universal *-algebra generated by the entries of a cubic matrix. More precisely $A_{h}(2)$ is the universal $*$-algebra presented by generators $\left(v_{i j}\right)_{1 \leq i, j \leq n}$ and relations:

(1) The matrix $v=\left(v_{i j}\right)$ is orthogonal.

(2) $v_{i j} v_{i k}=v_{i k} v_{k j}=0=v_{j i} v_{k i}=v_{k i} v_{j i}$ if $j \neq k$.

The Hopf $*$-algebras $\mathcal{R}\left(O_{-1}(2)\right)$ and $A_{h}(2)$ are isomorphic, via

$$
\left(\begin{array}{ll}
a_{11} & a_{12} \\
a_{21} & a_{22}
\end{array}\right) \longmapsto\left(\begin{array}{cc}
\frac{1}{\sqrt{2}} & \frac{-1}{\sqrt{2}} \\
\frac{1}{\sqrt{2}} & \frac{1}{\sqrt{2}}
\end{array}\right)\left(\begin{array}{ll}
v_{11} & v_{12} \\
v_{21} & v_{22}
\end{array}\right)\left(\begin{array}{cc}
\frac{1}{\sqrt{2}} & \frac{1}{\sqrt{2}} \\
\frac{-1}{\sqrt{2}} & \frac{1}{\sqrt{2}}
\end{array}\right)
$$

It is shown in [15] that there is an algebra isomorphism

$$
A_{h}(2) \simeq \mathbb{C}\left[\mathbb{Z}_{2} * \mathbb{Z}_{2}\right] \otimes \mathrm{C}\left[\mathbb{Z}_{2}\right]
$$

Using the group isomorphism $\mathbb{Z}_{2} * \mathbb{Z}_{2} \simeq D_{\infty}$, we get the following isomorphism, that will enable us to connect $A_{h}(2)$ with the algebras constructed by Masuoka in [28].

Lemma 7.1. Let $Z$ be the universal algebra presented by generators $z, z^{-1}, t, a$ and relations

$$
z z^{-1}=1=z^{-1} z, t^{2}=1=a^{2}, t z=z^{-1} t, a t=t a, a z=z a
$$


We have an algebra isomorphism

$$
\begin{aligned}
& A_{h}(2) \longrightarrow Z \\
&\left(\begin{array}{ll}
v_{11} & v_{12} \\
v_{21} & v_{22}
\end{array}\right) \longmapsto\left(\begin{array}{ll}
t \frac{(1+a)}{2} & t \frac{(1-a)}{2} \\
t z \frac{(1-a)}{2} & t z \frac{(1+a)}{2}
\end{array}\right)
\end{aligned}
$$

Proof. The map below defines the inverse isomorphism.

$$
t \mapsto v_{11}+v_{12}, z \mapsto v_{11} v_{22}+v_{12} v_{21}, a \mapsto v_{11}^{2}-v_{12}^{2}
$$

We now describe the Peter-Weyl decomposition of $A_{h}(2)$. We put $d=v_{11}^{2}-v_{12}^{2}$. This a group-like element satisfying $d^{2}=1$. For $k \in \mathbb{N}^{*}$, we define a comatrix subcoalgebra of $A_{h}(2)$ in the following way. For $k=2 m$ we put

$$
\left(\begin{array}{ll}
v_{11}^{[k]} & v_{12}^{[k]} \\
v_{21}^{[k]} & v_{22}^{[k]}
\end{array}\right)=\left(\begin{array}{ll}
\left(v_{11} v_{22}\right)^{m} & \left(v_{12} v_{21}\right)^{m} \\
\left(v_{21} v_{12}\right)^{m} & \left(v_{22} v_{11}\right)^{m}
\end{array}\right)
$$

For $k=2 m+1$, we put

$$
\left(\begin{array}{ll}
v_{11}^{[k]} & v_{12}^{[k]} \\
v_{21}^{[k]} & v_{22}^{[k]}
\end{array}\right)=\left(\begin{array}{ll}
\left(v_{11} v_{22}\right)^{m} v_{11} & \left(v_{12} v_{21}\right)^{m} v_{12} \\
\left(v_{21} v_{12}\right)^{m} v_{21} & \left(v_{22} v_{11}\right)^{m} v_{22}
\end{array}\right)
$$

We put $C(k)=\mathbb{C} v_{11}^{[k]} \oplus \mathbb{C} v_{12}^{[k]} \oplus \mathbb{C} v_{21}^{[k]} \oplus \mathbb{C} v_{22}^{[k]}$. The vector space $C(k)$ has dimension 4 by Lemma 7.1, and is a (simple) subcoalgebra of $A_{h}(2)$. An application of Lemma 7.1 yields the Peter-Weyl decomposition of $A_{h}(2)$.

Proposition 7.1. The Peter-Weyl decomposition of $A_{h}(2)$ is

$$
A_{h}(2)=\mathbb{C} 1 \oplus \mathbb{C} d \oplus\left(\oplus_{k \geq 1} C(k)\right)
$$

We now construct some finite-dimensional quotients of $A_{h}(2)$. For $m \in \mathbb{N}^{*}$ and $e= \pm 1$ we define $A(2 m, e)$ to be the quotient of $A_{h}(2)$ by the relations

$$
\left(v_{11} v_{22}\right)^{m}=\left(v_{22} v_{11}\right)^{m},\left(v_{12} v_{21}\right)^{m}=e\left(v_{21} v_{12}\right)^{m}
$$

Similarly, for $m \in \mathbb{N}$ and $e= \pm 1$, we define $A(2 m+1, e)$ to be the quotient of $A_{h}(2)$ by the relations

$$
\left(v_{11} v_{22}\right)^{m} v_{11}=\left(v_{22} v_{11}\right)^{m} v_{22},\left(v_{12} v_{21}\right)^{m} v_{12}=e\left(v_{21} v_{12}\right)^{m} v_{21}
$$

Proposition 7.2. The algebras $A(k, e)$ are Hopf *-algebra quotients of $A_{h}(2)$ for any $k \in \mathbb{N}^{*}$.

(1) For $k=1$, we have $A(1, e) \simeq \mathbb{C}[\mathrm{V}]$

(2) For $k=2$, the Hopf algebra $A(2,1)$ has dimension 8 and is isomorphic with $\mathrm{C}\left(D_{4}\right)$.

(3) For $k>2$, the Hopf algebra $A(k, 1)$ has dimension $4 k$ and is isomorphic with $\mathrm{C}\left(D_{2 k}^{\tau}\right)$, the function algebra on $D_{2 k}^{\tau}$, the unique non-trivial twist of the dihedral group $D_{2 k}$.

(4) For $k>2$, the Hopf algebra $A(k,-1)$ has dimension $4 k$ and is isomorphic with $\mathrm{C}\left(D C_{k}^{\tau}\right)$, the function algebra on $D C_{k}^{\tau}$, a non-trivial pseudotwist of the dicyclic group $D C_{k}$ considered by Masuoka [28], Nikshych [33], Suzuki [37] and Vainerman [38]. 
Proof. It is straightforward to check that $A(k, e)$ is indeed a Hopf $*$-algebra quotient of $A_{h}(2)$, and the assertion at $k=1$ is immediate since $A(1, e)$ is commutative. Also $A(2,1)$ is commutative, and hence is the function algebra on $D_{4}$.

Assume that $k>2$. Then by using Lemma 7.1 , we see that $A(k, 1)$ is isomorphic with the quotient of the algebra $Z$ of Lemma 7.1 by the relation $z^{k}=1$. Therefore $A(k, 1)$ is isomorphic with the algebra $\mathcal{A}_{4 k}$ considered by Masuoka (Definition 3.3 in [28]). This is a Hopf algebra isomorphism, and we conclude by Theorem 4.1 in [28] that $A(k, 1) \simeq \mathrm{C}\left(D_{2 k}^{\tau}\right)$.

Similarly, by using Lemma 7.1 , we see that $A(k,-1)$ is isomorphic with the quotient of the algebra $Z$ by the relation $z^{k}=a$. Therefore $A(k,-1)$ is isomorphic with the algebra $\mathcal{B}_{4 k}$ considered by Masuoka (Definition 3.3 in [28]). This is a Hopf algebra isomorphism. In $[33,38]$ the Hopf algebra $\mathcal{B}_{4 k}$ appears as a pseudo-twist of the dicyclic group, and this is why we put $\mathcal{B}_{4 k}=\mathrm{C}\left(D C_{k}^{\tau}\right)$.

We also will need the following technical lemma.

Lemma 7.2. Let $A$ be a Hopf *-algebra and let $w=\left(w_{i j}\right)$ in $M_{2}(A)$ be a unitary matrix such that $\Delta\left(w_{i j}\right)=\sum_{k} w_{i k} \otimes w_{k j}$ and $\varepsilon\left(w_{i j}\right)=\delta_{i j}$. Assume that $w_{i j} w_{i k}=$ $w_{i k} w_{i j}=0=w_{j i} w_{k i}=w_{k i} w_{j i}$ if $j \neq k$ and that there exists $F \in G L(2)$ such that the matrix $F w F^{-1}$ is diagonal.

(A) Assume that the matrix $w$ is orthogonal. Then one of the following happens.

(1) $w_{12}=0=w_{21}$ and $w_{11}^{2}=1=w_{22}^{2}$.

(2) $w_{11}=w_{22}$ and $w_{12}= \pm w_{21}$.

If moreover

$$
F w F^{-1}=\left(\begin{array}{ll}
1 & 0 \\
0 & *
\end{array}\right)
$$

Then according to the previous cases, one the following happens.

(1) $w_{11}=1$ or $w_{22}=1$.

(2) $w_{11}+w_{22}=1$ or $w_{11}+i w_{22}=1$

(B) Assume that the coefficients of the matrix $w$ pairwise commute and that $w_{11} w_{22}+$ $w_{12} w_{21}=1$. Then one of the following happens.

(1) $w_{12}=0=w_{21}$ and $w_{11} w_{22}=1=w_{22} w_{11}$.

(2) $w_{11}=w_{22}$ and $w_{12}=\lambda w_{21}$ for $\lambda \in \mathbb{C}$ with $|\lambda|=1$.

Proof. Let $F=\left(\begin{array}{ll}a & b \\ c & d\end{array}\right)$. Then if $F w F^{-1}$ is diagonal, we have the following equations

$$
\begin{aligned}
& a b\left(w_{11}-w_{22}\right)=a^{2} w_{12}-b^{2} w_{21} \\
& c d\left(w_{11}-w_{22}\right)=c^{2} w_{12}-d^{2} w_{21}
\end{aligned}
$$

Assume that we are in situation (A). If $b=0$, we have $a^{2} w_{12}=0$ and hence $w_{12}=0$. By applying the antipode we get $w_{21}=0$, and finally the orthogonality condition ensures that we are in case (1). The same reasoning holds if $a=0, c=0$ or $d=0$.

Assume now that the scalars $a, b, c, d$ are all nonzero. Then by multiplying the first equation in the proof by $w_{11}$ we get

$$
w_{11}^{2}=w_{11} w_{22}=w_{22} w_{11}=w_{22}^{2}
$$

and then

$$
w_{11}=w_{11}^{3}=w_{11} w_{22} w_{22}=w_{22}^{3}=w_{22} .
$$


We also get

$$
\left(a b^{-1}-c d^{-1}\right) w_{12}=\left(b a^{-1}-d c^{-1}\right) w_{21}
$$

and hence there exists $\lambda \in \mathbb{C}^{*}$ such that $w_{12}=\lambda w_{21}$. We then use the antipode to get $\lambda= \pm 1$, and we are in case (2).

In case (1), the group-like elements in the coalgebra generated by the entries of $w$ are $w_{11}$ and $w_{22}$, while in case $(2)$ they are $w_{11}+w_{22}$ and $w_{11}+i w_{22}$. The last assertion follows.

Let us assume now that condition (B) holds. If $b=0$, then $a^{2} w_{12}=0$ and $w_{12}=0=w_{21}$ : we are in case (1), and the same reasoning holds if $a, c$ or $d$ is zero.

Assume now that the scalars $a, b, c, d$ are all non-zero. Similarly to the previous case, we get $w_{12}=\lambda w_{21}$ for some $\lambda \in \mathbb{C}^{*}$, and by using the adjoint we get $|\lambda|=1$. Finally, since $S\left(w_{11}\right)=w_{22}$ and $S\left(w_{12}\right)=w_{12}$, by applying the antipode to the first equation at the beginning of the proof yields $w_{11}=w_{22}$ : we are in situation (2).

We now turn to classify the Hopf $*$-algebra quotients of $A_{h}(2)$. Let $L$ be a nontrivial Hopf $*$-algebra quotient of $A_{h}(2)$. For simplicity, the projections of the elements of $A_{h}(2)$ are denoted by the same symbol in $L$. The Peter-Weyl decomposition of $A_{h}(2)$ becomes a coalgebra sum

$$
L=\mathbb{C} 1+\mathbb{C} d+\left(\sum_{k \geq 1} C(k)\right)
$$

For $k \in \mathbb{N}^{*}$ the 2-dimensional corepresentation corresponding to the coalgebra $C(k)$ is denoted $V_{k}$, and $V_{k}$ is irreducible if and only if $\operatorname{dim} C(k)=4$.

Lemma 7.3. Assume that $d=v_{11}^{2}-v_{12}^{2}=1$ in L. Then $L$ is isomorphic with $\mathbb{C}\left[D_{\infty}\right]$, the group algebra of the infinite dihedral group or with $\mathbb{C}\left[D_{n}\right]$, the group algebra of the dihedral group of order $2 n$, for $n \in \mathbb{N}^{*}$.

Proof. Summing $d$ with $v_{11}^{2}+v_{12}^{2}=1$, we get $v_{11}^{2}=v_{22}^{2}=1$ and $v_{12}^{2}=v_{21}^{2}=0$. Since $v_{12}$ and $v_{21}$ are self-adjoint, we have $v_{12}=0=v_{21}$. We get a surjective Hopf *-algebra map $\mathbb{C}\left[D_{\infty}\right] \simeq \mathbb{C}\left[\mathbb{Z}_{2} * \mathbb{Z}_{2}\right] \rightarrow L$, and we conclude by using the classification of Hopf $*$-algebra quotients of $\mathbb{C}\left[D_{\infty}\right]$.

So we assume now that $d \neq 1$ in $L$, and that $L$ is not a quotient of $\mathbb{C}\left[D_{\infty}\right]$.

Lemma 7.4. Assume that the corepresentation $V_{k}$ is not irreducible for some $k \in$ $\mathbb{N}^{*}$. Then there exists a surjective Hopf $*$-algebra map $A(k, e) \rightarrow L$ for $e= \pm 1$, and in particular $\operatorname{dim} L$ divides $4 k$.

Proof. Assume first that $k=2 m+1$ is odd. Consider the matrix

$$
\left(\begin{array}{ll}
\left(v_{11} v_{22}\right)^{m} v_{11} & \left(v_{12} v_{21}\right)^{m} v_{12} \\
\left(v_{21} v_{12}\right)^{m} v_{21} & \left(v_{22} v_{11}\right)^{m} v_{22}
\end{array}\right)
$$

Then by the first part of Lemma 7.2 one of the following holds:

(1) $\left(v_{12} v_{21}\right)^{m} v_{12}=0=\left(v_{21} v_{12}\right)^{m} v_{21}$ and $\left(\left(v_{11} v_{22}\right)^{m} v_{11}\right)^{2}=1=\left(\left(v_{22} v_{11}\right)^{m} v_{22}\right)^{2}$.

(2) $\left(v_{11} v_{22}\right)^{m} v_{11}=\left(v_{22} v_{11}\right)^{m} v_{22}$ and $\left(v_{12} v_{21}\right)^{m} v_{12}= \pm\left(v_{12} v_{21}\right)^{m} v_{12}$.

In the first situation some direct computations give $v_{11}^{2}=1=v_{22}^{2}$ and $v_{12}=v_{21}=0$, contradicting the assumption on $L$. The second family of relations yields a surjective Hopf $*$-algebra map $A(k, e) \rightarrow L$. 
Similarly, if $k=2 m$ is even, we consider the matrix

$$
\left(\begin{array}{ll}
\left(v_{11} v_{22}\right)^{m} & \left(v_{12} v_{21}\right)^{m} \\
\left(v_{21} v_{12}\right)^{m} & \left(v_{22} v_{11}\right)^{m}
\end{array}\right)
$$

By the second part of Lemma 7.2, one of the following holds:

(1) $\left(v_{12} v_{21}\right)^{m}=0=\left(v_{21} v_{12}\right)^{m}$ and $\left(\left(v_{11} v_{22}\right)^{m}\right)^{2}=1=\left(\left(v_{22} v_{11}\right)^{m}\right)^{2}$.

(2) $\left(v_{11} v_{22}\right)^{m}=\left(v_{22} v_{11}\right)^{m}$ and $\left(v_{12} v_{21}\right)^{m}=\lambda\left(v_{21} v_{12}\right)^{m}$.

Similarly to the previous case, the first family of relations cannot hold. If $\lambda= \pm 1$ in the second family of relations, we have our result. Otherwise we have

$$
\begin{aligned}
\left(v_{12} v_{21}\right)^{m} v_{21} & =\lambda\left(v_{21} v_{12}\right)^{m} v_{21} \\
\Rightarrow\left(v_{12} v_{21}\right)^{m-1} v_{12} & =\lambda v_{21}\left(v_{12} v_{21}\right)^{m}=\lambda^{2} v_{21}\left(v_{21} v_{12}\right)^{m} \\
& =\lambda^{2} v_{21}^{2}\left(v_{12} v_{21}\right)^{m-1} v_{12}=\lambda^{2}\left(v_{12} v_{21}\right)^{m-1} v_{12}
\end{aligned}
$$

If $\lambda^{2} \neq 1$, we have then

$$
\left(v_{12} v_{21}\right)^{m-1} v_{12}=0=\left(v_{12} v_{21}\right)^{m}=\left(v_{21} v_{12}\right)^{m}
$$

and hence $\left(v_{12} v_{21}\right)^{m}= \pm\left(v_{21} v_{12}\right)^{m}$ : we have our result.

The last assertion follows from the Nichols-Zoeller theorem.

This last lemma before the classification of the Hopf $*$-algebra quotients of $A_{h}(2)$ uses the following fusion rules of $A_{h}(2)$ (see [15], these are the same as the ones of $O(2))$ :

$$
V_{i} \otimes V_{j} \simeq V_{i+j} \oplus V_{|i-j|} \text { if } i \neq j \in \mathbb{N}^{*}
$$

$V_{i} \otimes V_{i} \simeq \mathbb{C} \oplus \mathbb{C} d \oplus V_{2 i} ; \mathbb{C} d \otimes \mathbb{C} d \simeq \mathbb{C} ; V_{i} \otimes \mathbb{C} d \simeq \mathbb{C} d \otimes V_{i} \simeq V_{i}, i \in \mathbb{N}^{*}$.

Lemma 7.5. Let $j>2$ and assume that

(1) The corepresentations $V_{1}, \ldots, V_{j-1}$ of $L$ are irreducible and pairwise nonequivalent.

(2) For $l \in\{1, \ldots, j-1\}$, we have $V_{l} \simeq V_{j}$.

Then $j \neq l+1$ and $L \simeq A(l+j, e)$ for $e= \pm 1$.

Proof. We have $V_{l} \simeq V_{j}$ for $l<j$ and hence

$$
V_{l} \otimes V_{j} \simeq V_{j-l} \oplus V_{l+j} \simeq V_{l} \otimes V_{l} \simeq \mathbb{C} \oplus \mathbb{C} d \oplus V_{2 l}
$$

Hence $V_{l+j}$ is not irreducible, with $V_{l+j} \simeq \mathbb{C} \oplus \mathbb{C} d$, while $V_{2 l}$ is irreducible with $V_{2 l} \simeq$ $V_{j-l}$. By the previous lemma we have a surjective Hopf $*$-algebra map $A(l+j, e) \rightarrow L$ and $\operatorname{dim} L$ divides $4(l+j)$. A counting argument using the first assumption shows that $\operatorname{dim} L \geq 4(j-1)+2=4 j-2$.

Assume that $j-l>1$. Then $4 j-2>4(l+j) / 2$, and hence $\operatorname{dim} L>4(l+j) / 2$. We conclude that $\operatorname{dim} L=4(l+j)$ and that $L \simeq A(l+j, e)$.

Assume now that $j=l+1$. Then $V_{2 l+1} \simeq \mathbb{C} \oplus \mathbb{C} d$ and $V_{2 l}$ is irreducible. By Lemma 7.2 , we have $\left(v_{11} v_{22}\right)^{l} v_{11}=1$, or $\left(v_{22} v_{11}\right)^{l} v_{11}=1$, or $\left(v_{11} v_{22}\right)^{l} v_{11}+\left(v_{22} v_{11}\right)^{l} v_{22}=1$ or $\left(v_{11} v_{22}\right)^{l} v_{11}+i\left(v_{22} v_{11}\right)^{l} v_{22}=1$. If for example $\left(v_{11} v_{22}\right)^{l} v_{11}=1$, we get, multiplying by $v_{11}$, that $\left(v_{11} v_{22}\right)^{l}=\left(v_{22} v_{11}\right)^{l}=v_{11}$, which contradicts the irreducibility of $V_{2 l}$. The other cases are treated similarly, and we are done. 
We are now ready to complete the classification of the compact quantum subgroups of $O_{-1}(2)$.

Theorem 7.1. Any non-trivial Hopf *-algebra quotient of $A_{h}(2)$ is isomorphic to one of the following Hopf *-algebras.

(1) $\mathbb{C}\left[D_{\infty}\right]$, the group algebra of the infinite dihedral group.

(2) $\mathbb{C}\left[D_{n}\right]$, the group algebra of the dihedral group of order $2 n, n \in \mathbb{N}^{*}$.

(3) $A(k, e), k \in \mathbb{N}^{*}, e= \pm 1$.

Proof. Let $L$ be a non-trivial Hopf $*$-algebra quotient of $A_{h}(2)$. We can assume that $L$ is not a quotient of $\mathbb{C}\left[D_{\infty}\right]$, and that $d \neq 1$. Then the comodule $V_{1}$ is irreducible. If all the comodules $V_{i}$ are irreducible and pairwise non-equivalent, it is clear that $L \simeq A_{h}(2)$. Hence we can consider a family of pairwise non-equivalent irreducible comodules $V_{1}, \ldots, V_{j-1}(j>1)$ such that either

(1) $V_{j}$ is irreducible and there exists $l \in\{1, \ldots, j-1\}$ with $V_{l} \simeq V_{j}$,

(2) $V_{j}$ is not irreducible.

The first situation is the one of the previous lemma, and we conclude that $L \simeq$ $A(l+j, e)$.

In the second situation, we have, by Lemma 7.4, a surjective Hopf $*$-algebra map $A(j, e) \rightarrow L$, and $\operatorname{dim} L \mid 4 j$. But by the assumption $\operatorname{dim} L \geq 4 j-2$, and hence we conclude that $\operatorname{dim} L=4 j$ and that $L \simeq A(j, e)$.

\section{Strategy For the ADE Classifichtion: ALGEBraiC InVARIANts}

The proof of the main result, Theorem 1.1, is now complete. In the rest of this paper we describe an ADE type classification of the quantum subgroups we have found. This section is devoted to the description of our strategy.

The ADE classification, i.e. McKay correspondence, for (compact) subgroups of $S O(3)$ is obtained as follows. Starting from such a subgroup $G \subset S O(3)$, we consider its double cover $G^{\prime} \subset S U(2)$, and associate to it its McKay graph [31] to obtain an ADE graph. Here $\mathcal{Q}_{4} \simeq S O_{-1}(3)$ does not have such a double cover, and we do not know a direct way to associate a graph to a subgroup of $\mathcal{Q}_{4}$. Therefore we proceed as follows.

We consider the following two types of objects.

(1) Quantum groups acting on 4 points.

(2) ADE type graphs.

To any such object $X$ (a quantum group or a graph), we associate a sequence of integers $\left(c_{n}\right)$, or equivalently a probability measure on the circle (the circular measure), that we call the algebraic invariants of $X$. Then we say that a quantum group and a graph correspond to each other if they have the same algebraic invariants.

This might look a bit artificial, but the following facts support our strategy.

(1) The classical McKay correspondence might be described in such a way, see Section 11.

(2) When the quantum group acts ergodically on $\mathbb{C}^{4}$, one can associate a subfactor of index 4 to this data [3]. The principal graph of the subfactor is the ADE graph we find. 
Let us begin with the algebraic invariants of a quantum permutation group $\mathcal{G} \subset$ $\mathcal{Q}_{4}$. These depend not only on $\mathcal{G}$, but also on its embedding into $\mathcal{Q}_{4}$. These invariants come from the spectral theory of the character of the fundamental representation of $\mathcal{G}$, on the space $\mathbb{C}^{4}$. They are best introduced in the following way.

Definition 8.1. The algebraic invariants of a quantum subgroup $\mathcal{G} \subset \mathcal{Q}_{4}$ are:

(1) The multiplicity $c_{k}$. This is the number of copies of 1 into $u^{\otimes k}$.

(2) The spectral measure $\mu$. This is the measure having $c_{k}$ as moments.

(3) The circular measure $\varepsilon$. This is the pullback of $\mu$ via $\varphi(q)=(q+\bar{q})^{2}$.

In this definition $\mu, \varepsilon$ are probability measures on the real line, and on the unit circle. The support of $\mu$ being contained in $[0,4]$ and the map $\varphi$ being surjective from the unit circle to $[0,4]$, the measure $\varepsilon$ is well-defined, by distributing the weight at $x \in[0,4]$ uniformly among its preimages $q=\varphi^{-1}(x)$. See [4], [9].

We use the following notations:

(1) $d$ is the uniform measure on the unit circle.

(2) $d_{n}$ is the uniform measure on $2 n$-roots of 1 .

(3) $d_{n}^{\prime}$ is the uniform measure on odd $4 n$-roots of 1 .

(4) $e_{n}=d_{1}^{\prime}$ if $n$ is even and $e_{n}=d_{2}^{\prime}$ if $n$ is odd.

(5) For $s \in[0,4], \gamma_{s}$ is the pullback of $\delta_{s}$, the Dirac mass at $s$, via $\varphi(q)=(q+\bar{q})^{2}$.

We let $\alpha(q)=2 \operatorname{Im}(q)^{2}$. This is the pullback via $\varphi$ of the free Poisson density $(2 \pi)^{-1} \sqrt{4 x^{-1}-1}$ of Marchenko-Pastur [29] and Voiculescu [39].

We discuss now algebraic invariants of graphs. The interest in these invariants might seem less clear than in the quantum group case. The idea is that these are related to the quantum group or subfactor ones via the representation-theoretic notion of principal graph. See $[9,25]$.

Definition 8.2. The algebraic invariants of a rooted graph of norm $\leq 2$ are:

(1) The loop number $c_{k}$. This is the number of $2 k$-loops based at the root.

(2) The spectral measure $\mu$. This is the measure having $c_{k}$ as moments.

(3) The circular measure $\varepsilon$. This is the pullback of $\mu$ via $\varphi(q)=(q+\bar{q})^{2}$.

The comment after previous definition applies as well to this situation, and shows that $\varepsilon$ is indeed a probability measure on the unit circle. Here the fact that $\mu$ is supported by $[0,4]$ comes from the fact that the norm of the graph is $\leq 2$.

In fact, $\mu$ is the spectral measure of the square of the adjacency matrix of the graph, and the norm $\leq 2$ condition means that this adjacency matrix is supported by $[-2,2]$, hence its square is supported by $[0,4]$. See $[9]$ for details.

The remaining sections are devoted to the description of the circular measures of quantum permutation groups and ADE graphs. For this purpose it is convenient to divide the quantum subgroups of $\mathcal{Q}_{4}$ in several classes, which leads to the following reformulation of Theorem 1.1.

Theorem 8.1. The quantum subgroups of $\mathcal{Q}_{4}$ are of three types.

(1) Classical: $\mathbb{Z}_{1}, \mathbb{Z}_{2}, \mathbb{Z}_{3}, \mathbb{Z}_{4}, V, S_{3}, D_{4}, A_{4}, S_{4}$.

(2) Quantum, single: $S_{4}^{\tau}, A_{5}^{\tau}, \widehat{D}_{\infty}, O_{-1}(2), \mathcal{Q}_{4}$.

(3) Quantum, series: $\widehat{D}_{n}, D_{2 n}^{\tau}$ with $n \geq 3, D C_{n}^{\tau}$ with $n \geq 2$. 
We begin by treating the case of the subgroups of $S_{4}$ in the next section. In Section 10, we describe the circular measures of the extended Coxeter-Dynkin graphs (taken from [9]), plus two other graphs. In Section 11, we treat the remaining quantum subgroups, using a reformulation of McKay correspondence at the level of algebraic invariants, and Section 12 is devoted to the final classification tables.

\section{Algebraic invariants of subgroups of $S_{4}$}

The subgroups of $S_{4}$ are $\mathbb{Z}_{1}, \mathbb{Z}_{2}, \mathbb{Z}_{3}, \mathbb{Z}_{4}, V, S_{3}, D_{4}, A_{4}, S_{4}$. In this section we discuss the algebraic invariants of these groups.

Before starting, we have to discuss a quite undexpected issue.

Definition 9.1. The 2-element groups acting on 4 points are as follows.

(1) $D_{1}$ : here the non-trivial element acts with 2 fixed points.

(2) $\mathbb{Z}_{2}$ : here the non-trivial element acts without fixed point.

The Klein groups acting on 4 points are as follows.

(1) $D_{2}$ : here the non-trivial elements acts without fixed points.

(2) $V$ : here there are 2 non-trivial elements having 2 fixed points.

The group $D_{1}$ is to be added to the above list, as a 10-th classical subgroup of $S_{4}$. Observe that the notation $D_{1}$ is in agreement with the isomorphisms for small dihedral groups, which are $D_{1} \simeq \mathbb{Z}_{2}, D_{2} \simeq V, D_{3} \simeq S_{3}$.

The point is that the algebraic invariants of a quantum permutation group $\mathcal{G} \subset \mathcal{Q}_{4}$ depend not only on $\mathcal{G}$, but also on its embedding into $\mathcal{Q}_{4}$. In fact $D_{2}$ has the same algebraic invariants as $\mathbb{Z}_{4}$, so we will ignore it.

The computation for symmetric groups leads to the following formulae, the notations for circular measures being the ones of the previous section.

Theorem 9.1. The circular measures of subgroups $G \subset S_{4}$ are as follows.

(1) For $\mathbb{Z}_{1}, \mathbb{Z}_{2}, \mathbb{Z}_{3}, V$ we have $\varepsilon=d_{n}$ with $n=1,2,3,4$.

(2) For $D_{1}, \mathbb{Z}_{4}, S_{3}, D_{4}$ we have $\varepsilon=\left(e_{n}+d_{n}\right) / 2$ with $n=1,2,3,4$.

(3) For $A_{4}, S_{4}$ we have $\varepsilon=\alpha d_{n}+\left(d_{n-1}-d_{n}\right) / 2$ with $n=3,4$.

Proof. For $G \subset S_{4}$ we denote by $m_{s}$ the number of elements in $G$ having $s$ fixed points. Let also $m=|G|$. Then the invariants of $G$ are:

$$
\begin{aligned}
c_{k} & =\frac{1}{m} \sum_{s=0}^{4} m_{s} s^{k} \\
\mu & =\frac{1}{m} \sum_{s=0}^{4} m_{s} \delta_{s} \\
\varepsilon & =\frac{1}{m} \sum_{s=0}^{4} m_{s} \gamma_{s}
\end{aligned}
$$

These formulae are all equivalent, and the first two ones are valid in fact for any subgroup $G \subset S_{n}$, with 4 replaced of course by $n$. See [4] for details. 
We have $m_{3}=0$ and $m_{4}=1$, so all invariants can be expressed in terms of the numbers $m_{0}, m_{1}, m_{2}$ and $m=m_{0}+m_{1}+m_{2}+1$ :

$$
\begin{aligned}
c_{k} & =\frac{1}{m}\left(m_{1}+m_{2} 2^{k}+4^{k}\right) \\
\mu & =\frac{1}{m}\left(m_{0} \delta_{0}+m_{1} \delta_{1}+m_{2} \delta_{2}+\delta_{4}\right) \\
\varepsilon & =\frac{1}{m}\left(m_{0} \gamma_{0}+m_{1} \gamma_{1}+m_{2} \gamma_{2}+\gamma_{4}\right)
\end{aligned}
$$

The relevant measures $\gamma_{s}$ are as follows:

$$
\begin{aligned}
\gamma_{0} & =d_{1}^{\prime} \\
\gamma_{1} & =\left(3 d_{3}-d_{1}\right) / 2 \\
\gamma_{2} & =d_{2}^{\prime} \\
\gamma_{4} & =d_{1}
\end{aligned}
$$

Thus we have the following formula for the circular measure:

$$
\varepsilon=\frac{1}{m}\left(m_{0} d_{1}^{\prime}+\frac{m_{1}}{2}\left(3 d_{3}-d_{1}\right)+m_{2} d_{2}^{\prime}+d_{1}\right)
$$

The numbers $m_{0}, m_{1}, m_{2}$ and $m=m_{0}+m_{1}+m_{2}+1$ are as follows:

\begin{tabular}{|l|l|l|l|l|l|l|l|l|l|l|}
\hline & $\mathbb{Z}_{1}$ & $\mathbb{Z}_{2}$ & $\mathbb{Z}_{3}$ & $V$ & $D_{1}$ & $\mathbb{Z}_{4}$ & $S_{3}$ & $D_{4}$ & $A_{4}$ & $S_{4}$ \\
\hline$m_{0}$ & 0 & 1 & 0 & 1 & 0 & 3 & 0 & 5 & 3 & 9 \\
\hline$m_{1}$ & 0 & 0 & 2 & 0 & 0 & 0 & 2 & 0 & 8 & 8 \\
\hline$m_{2}$ & 0 & 0 & 0 & 2 & 1 & 0 & 3 & 2 & 0 & 6 \\
\hline$m$ & 1 & 2 & 3 & 4 & 2 & 4 & 6 & 8 & 12 & 24 \\
\hline
\end{tabular}

Observe that $V$ acts with $m_{0}=0$. The other action of $V$, with $m_{0}=3$, gives the same algebraic invariants as $\mathbb{Z}_{4}$, so we will not consider it.

We are now in position of computing the circular measures. We will use many times the formula $d_{n}+d_{n}^{\prime}=2 d_{2 n}$.

For the groups $\mathbb{Z}_{1}, \mathbb{Z}_{2}, \mathbb{Z}_{3}, V, D_{1}, \mathbb{Z}_{4}, S_{3}, D_{4}$ we immediatly get the measures in the statement.

For $A_{4}$ we have the following preliminary computation:

$$
\begin{aligned}
\varepsilon & =\left(3 d_{1}^{\prime}+4\left(3 d_{3}-d_{1}\right)+d_{1}\right) / 12 \\
& =\left(3 d_{1}^{\prime}-3 d_{1}+12 d_{3}\right) / 12 \\
& =\left(d_{1}^{\prime}-d_{1}+4 d_{3}\right) / 4 \\
& =d_{3}+\left(d_{1}^{\prime}-d_{1}\right) / 4
\end{aligned}
$$

For $S_{4}$ we have the following preliminary computation:

$$
\begin{aligned}
\varepsilon & =\left(9 d_{1}^{\prime}+4\left(3 d_{3}-d_{1}\right)+6 d_{2}^{\prime}+d_{1}\right) / 24 \\
& =\left(9 d_{1}^{\prime}-3 d_{1}+6 d_{2}^{\prime}+12 d_{3}\right) / 24 \\
& =\left(6 d_{2}+6 d_{1}^{\prime}-6 d_{1}+6 d_{2}^{\prime}+12 d_{3}\right) / 24 \\
& =\left(12 d_{4}+6 d_{1}^{\prime}-6 d_{1}+12 d_{3}\right) / 24 \\
& =\left(d_{3}+d_{4}\right) / 2+\left(d_{1}^{\prime}-d_{1}\right) / 4
\end{aligned}
$$


Consider now the fundamental density $\alpha(q)=2 \operatorname{Im}(q)^{2}$. The measure $\alpha d_{3}$ is uniformly supported by the imaginary 6 -roots, so we have $\alpha d_{3}=\gamma_{1}$. We get:

$$
\begin{aligned}
\alpha d_{3}+\left(d_{2}-d_{3}\right) / 2 & =\gamma_{1}+\left(d_{2}-d_{3}\right) / 3 \\
& =\left(3 d_{3}-d_{1}\right) / 2+\left(d_{2}-d_{3}\right) / 2 \\
& =d_{3}+\left(d_{2}-d_{1}\right) / 2 \\
& =d_{3}+\left(d_{1}^{\prime}-d_{1}\right) / 4
\end{aligned}
$$

Also, regarding the measure $\alpha d_{4}$, this has density $1 / 4$ at $\pm i$, and density $1 / 8$ at the odd 8-roots. Thus we have $\alpha d_{4}=\left(\gamma_{0}+\gamma_{2}\right) / 2$, which gives:

$$
\begin{aligned}
\alpha d_{4}+\left(d_{3}-d_{4}\right) / 2 & =\left(\gamma_{0}+\gamma_{2}\right) / 2+\left(d_{3}-d_{4}\right) / 2 \\
& =\left(d_{1}^{\prime}+d_{2}^{\prime}\right) / 2+\left(d_{3}-d_{4}\right) / 2 \\
& =\left(2 d_{2}-d_{1}+2 d_{4}-d_{2}\right) / 2+\left(d_{3}-d_{4}\right) / 2 \\
& =\left(d_{3}+d_{4}\right) / 2+\left(d_{2}-d_{1}\right) / 2 \\
& =\left(d_{3}+d_{4}\right) / 2+\left(d_{1}^{\prime}-d_{1}\right) / 4
\end{aligned}
$$

This gives the formulae for $A_{4}, S_{4}$ in the statement.

\section{Algebraic invariants of ADE type graphs}

We describe now the algebraic invariants of ADE type graphs. The extended Coxeter-Dynkin diagrams are as follows:
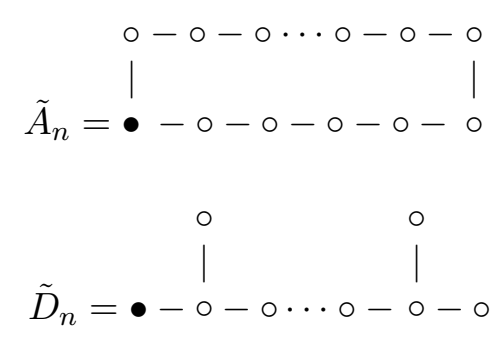

$$
\begin{gathered}
\stackrel{\circ}{\mid} \circ \\
\tilde{E}_{6}=\bullet-\circ-\circ-\circ-\circ
\end{gathered}
$$

$$
\begin{gathered}
\tilde{E}_{7}=\bullet-\circ-\circ-\circ-\circ-\circ-\circ \mid \\
\tilde{E}_{8}=\bullet-\circ-\circ-\circ-\circ-\circ-\circ-\circ
\end{gathered}
$$

$$
A_{\infty}=\bullet-\circ-\circ-\circ \cdots
$$

Here the graphs $\tilde{A}_{n}$ with $n \geq 1$ and $\tilde{D}_{n}$ with $n \geq 4$ have $n+1$ vertices each. Observe that the graphs $\tilde{E}_{n}$ with $n=6,7,8$ have $n+1$ vertices each as well. 
The first AD graphs are by definition as follows:

$$
\begin{array}{cc}
\| & \circ \\
\tilde{A}_{1}=\bullet & \backslash / \\
\tilde{D}_{4}=\bullet-\circ-\circ
\end{array}
$$

The graph $\tilde{A}_{n}$ as drawn has an even number of vertices. This will be in fact the case: we will only be concerned with graphs of type $\tilde{A}_{2 n-1}$, having $2 n$ vertices.

We use as well the following graphs, that we call type DE ghost graphs:

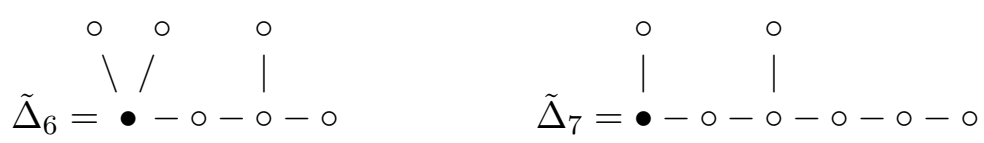

Here the subscript $n=6,7$ comes from the number of vertices, which is $n+1$ as usual. Observe that these two graphs are in fact bad-rooted versions of $\tilde{D}_{6}, \tilde{E}_{7}$. More comments about them will be given in the end of this section.

The pictures suggest that we have convergences $\tilde{A}_{n} \rightarrow A_{-\infty, \infty}$ and $\tilde{D}_{n} \rightarrow D_{\infty}$ (and, in some virtual sense, $\tilde{E}_{n} \rightarrow A_{\infty}$ ). The following result from [9] shows that indeed it is so, at level of circular measures. Moreover, the measures for each series can be recaptured by discretizing the limiting measure at certain roots of unity:

Theorem 10.1. The circular measures of ADE graphs are as follows, where the numbers $t, s$ are given by $t=1,3$ for $n=6,7$ and $s=3,4,6$ for $n=6,7,8$.

(1) For $\tilde{A}_{2 n-1}$ we have $\varepsilon=d_{n}$.

(2) For $A_{-\infty, \infty}$ we have $\varepsilon=d$.

(3) For $\tilde{D}_{n+2}$ we have $\varepsilon=\left(d_{1}^{\prime}+d_{n}\right) / 2$.

(4) For $D_{\infty}$ we have $\varepsilon=\left(d_{1}^{\prime}+d\right) / 2$.

(5) For $\tilde{\Delta}_{n}$ we have $\varepsilon=\left(d_{2}^{\prime}+d_{t}\right) / 2$.

(6) For $\tilde{E}_{n}$ we have $\varepsilon=\alpha d_{s}+\left(d_{s-1}-d_{s}\right) / 2$.

(7) For $A_{\infty}$ we have $\varepsilon=\alpha d$.

Proof. As already mentioned, the result is from [9]. We just have to prove the ghost formula (5), not considered in there.

For $\tilde{\Delta}_{6}$ we denote by $c_{k}, d_{k}$ the number of $2 k$-paths from the root $c$ to itself, and to the second vertex at right $d$, as indicated below:

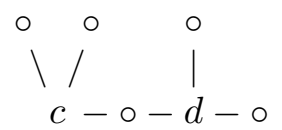

Since $c, d$ are at even distances on the graph, the $2 k$-paths counted by $c_{k}, d_{k}$ can be computed recursively. The equations are as follows:

$$
\begin{aligned}
c_{k+1} & =3 c_{k}+d_{k} \\
d_{k+1} & =c_{k}+3 d_{k}
\end{aligned}
$$

The starting values being $c_{1}=3, d_{1}=1$, we have the following solution:

$$
\begin{aligned}
& c_{k}=\left(4^{k}+2^{k}\right) / 2 \\
& d_{k}=\left(4^{k}-2^{k}\right) / 2
\end{aligned}
$$


On the other hand, the formula of $c_{k}$ is the one for multiplicities for the group $D_{1}$, from previous proof. Thus the algebraic invariants of $\tilde{\Delta}_{6}$ are those of $D_{1}$ computed in previous theorem, and in particular we have $\varepsilon=\left(d_{2}^{\prime}+d_{1}\right) / 2$ as claimed.

For $\tilde{\Delta}_{7}$ we denote by $c_{k}, d_{k}, e_{k}$ the number of $2 k$-paths from the root $c$ to itself, to the second vertex at right $d$, and to the fourth vertex at right $e$ :

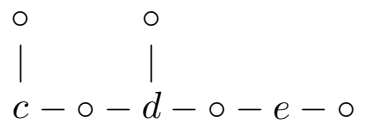

Since $c, d, e$ are at even distances on the graph, the $2 k$-paths counted by $c_{k}, d_{k}, e_{k}$ can be computed recursively. The equations are as follows:

$$
\begin{aligned}
c_{k+1} & =2 c_{k}+d_{k} \\
d_{k+1} & =c_{k}+3 d_{k}+e_{k} \\
e_{k+1} & =d_{k}+2 e_{k}
\end{aligned}
$$

The starting values being $c_{1}=2, d_{1}=1, e_{1}=0$, we get $c_{2}=5, c_{3}=15, c_{4}=51$ and so on. The general formula can be deduced by solving the system, and is:

$$
c_{k}=\frac{1}{6}\left(2+3 \cdot 2^{k}+4^{k}\right)
$$

On the other hand, the coefficients $m_{1}=2, m_{2}=3$ and $m=6$ appearing in this formula are nothing but the $m_{1}, m_{2}, m$ numbers for the group $S_{3}$, from previous proof. Thus the algebraic invariants of $\tilde{\Delta}_{7}$ are those of $S_{3}$ computed in previous theorem, and in particular we have $\varepsilon=\left(d_{2}^{\prime}+d_{3}\right) / 2$, and we are done.

Theorem 10.2. We have the classification table

\begin{tabular}{|l|l|l|l|l|l|l|l|l|l|}
\hline$\tilde{A}_{1}$ & $\tilde{A}_{3}$ & $\tilde{A}_{5}$ & $\tilde{A}_{7}$ & $\tilde{D}_{4}$ & $\tilde{D}_{6}$ & $\tilde{\Delta}_{6}$ & $\tilde{\Delta}_{7}$ & $\tilde{E}_{6}$ & $\tilde{E}_{7}$ \\
\hline $\mathbb{Z}_{1}$ & $\mathbb{Z}_{2}$ & $\mathbb{Z}_{3}$ & $V$ & $\mathbb{Z}_{4}$ & $D_{4}$ & $D_{1}$ & $S_{3}$ & $A_{4}$ & $S_{4}$ \\
\hline
\end{tabular}

making correspond graphs and groups having the same algebraic invariants.

Proof. This follows from the previous two results.

We end the section with some comments on the following problem: are $\tilde{\Delta}_{6}, \tilde{\Delta}_{7}$ part of some bigger series of graphs? That is, we would like to have some natural series of graphs $X_{n}$, having the property that $\tilde{\Delta}_{6}, \tilde{\Delta}_{7}$ are specializations of it.

There are two possible solutions, coming from the results that we have so far:

(1) $X_{n}$ is the graph having spectral measure $\left(e_{n}+d_{n}\right) / 2$.

(2) $X_{n}$ is the graph having spectral measure $\left(d_{2}^{\prime}+d_{n}\right) / 2$.

The first interpretation, coming from results in previous section, is not the good one: now we know from the general ADE formulae that at even levels we will get usual dihedral graphs, so this series $X_{n}$ is quite unnatural.

The second interpretation is probably the good one, and we will discuss it here in detail. First, we restrict attention to the $\operatorname{supp}(\mu) \subset \mathbb{N}$ case. 
Proposition 10.1. There are 5 measures of type $\varepsilon_{n}=\left(d_{2}^{\prime}+d_{n}\right) / 2$ which appear as linear combinations of $\gamma_{s}$ with $s=0,1,2,3,4$, namely:

$$
\begin{aligned}
& \varepsilon_{1}=\left(\gamma_{2}+\gamma_{4}\right) / 2 \\
& \varepsilon_{2}=\left(\gamma_{0}+2 \gamma_{2}+\gamma_{4}\right) / 4 \\
& \varepsilon_{3}=\left(2 \gamma_{1}+3 \gamma_{2}+\gamma_{4}\right) / 6 \\
& \varepsilon_{4}=\left(\gamma_{0}+6 \gamma_{2}+\gamma_{4}\right) / 8 \\
& \varepsilon_{6}=\left(\gamma_{0}+2 \gamma_{1}+6 \gamma_{2}+2 \gamma_{3}+\gamma_{4}\right) / 12
\end{aligned}
$$

Proof. First we note that there are 5 measures of type $d_{n}$ which appear as linear combinations of $\gamma_{s}$ with $s=0,1,2,3,4$, namely those corresponding to $n=1,2,3,4,6$, given by the following formulae:

$$
\begin{aligned}
d_{1} & =\gamma_{4} \\
d_{2} & =\left(\gamma_{0}+\gamma_{4}\right) / 2 \\
d_{3} & =\left(2 \gamma_{1}+\gamma_{4}\right) / 3 \\
d_{4} & =\left(\gamma_{0}+2 \gamma_{2}+\gamma_{4}\right) / 4 \\
d_{6} & =\left(\gamma_{0}+2 \gamma_{1}+2 \gamma_{3}+\gamma_{4}\right) / 6
\end{aligned}
$$

The formulae in the proposition are deduced by making averages between $d_{2}^{\prime}=\gamma_{2}$ and the measures $d_{n}$. The uniqueness assertion is clear.

We know that the measures $\varepsilon_{1}, \varepsilon_{2}, \varepsilon_{3}$ correspond to the groups $D_{1}, V, S_{3}$ and to the graphs $\tilde{\Delta}_{6}, \tilde{A}_{7}, \tilde{\Delta}_{7}$.

For the remaining measures $\varepsilon_{4}, \varepsilon_{6}$, we have a problem with graphs. For $\varepsilon_{4}$, the corresponding loop numbers are $c_{k}=\left(6 \cdot 2^{k}+4^{k}\right) / 8$, leading to the following system of equations:

$$
\begin{aligned}
c_{k+1} & =2 c_{k}+d_{k} \\
d_{k+1} & =c_{k}+2 d_{k}+3 e_{k} \\
e_{k+1} & =d_{k}+2 e_{k}
\end{aligned}
$$

The starting values here are $c_{1}=2, d_{1}=2, e_{1}=0$, and the first three loop numbers are $c_{1}=2, c_{2}=5, c_{3}=14$. These numeric values, along with the system itself, lead to the conclusion that the graph must look as follows:

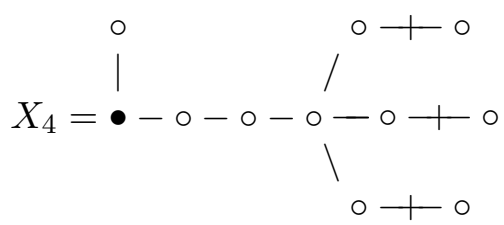

Here the three special edges at right are negative, in the sense that a loop through any of them adds -1 to the number of loops which are counted. The fact that this graph gives the above $c_{d}, d_{k}, e_{k}$ numbers follows by using the method in proof of Theorem 10.1. This is the simplest picture of $X_{4}$ that we were able to find.

The situation for the remaining measure $\varepsilon_{6}$ is quite similar. As a conclusion, $\tilde{\Delta}_{6}$, $\tilde{\Delta}_{7}$ are either exceptional, or are part of a series of non-standard graphs. 


\section{Quantum SubGRoups AND ClASSifiCATION TABLES}

We discuss now the non-classical subgroups of $\mathcal{Q}_{4}$. Most of them appear as twists of subgroups of $S O(3)$, so we first take a look at these subgroups. These are:

(1) The cyclic groups $\mathbb{Z}_{n}$ and the dihedral groups $D_{n}$.

(2) Their limits $S O(2)$ and $O(2)$, plus $S O(3)$ itself.

(3) The exceptional groups $A_{4}, S_{4}, A_{5}$.

The McKay correspondence [31] provides us with an ADE classification of these subgroups. For the purposes of this paper, we will need a direct proof of this result, in terms of algebraic invariants similar to those considered in previous sections.

We use the canonical identification $S O(3) \simeq P U(2)$, which endows $S O(3)$ with a fundamental representation $\pi$, on the Hilbert space $M_{2}(\mathbb{C}) \simeq \mathbb{C}^{4}$. We have $\pi=1+\rho$, where $\rho$ is the canonical representation on $\mathbb{C}^{3}$, coming from that on $\mathbb{R}^{3}$.

Definition 11.1. The algebraic invariants of a subgroup $G \subset S O(3)$ are:

(1) The multiplicity $c_{k}$. This is the number of copies of 1 into $\pi^{\otimes k}$.

(2) The spectral measure $\mu$. This is the measure having $c_{k}$ as moments.

(3) The circular measure $\varepsilon$. This is the pullback of $\mu$ via $\varphi(q)=(q+\bar{q})^{2}$.

In this definition $\mu$ and $\varepsilon$ are as usual probability measures on the real line, and on the unit circle.

With these notations, the ADE classification of subgroups of $S O(3)$ coming from the McKay correspondence can be stated in the following way:

Theorem 11.1. We have the classification table

\begin{tabular}{|l|l|l|l|l|l|l|l|}
\hline$\tilde{A}_{2 n-1}$ & $A_{-\infty, \infty}$ & $A_{\infty}$ & $\tilde{D}_{n+2}$ & $D_{\infty}$ & $\tilde{E}_{6}$ & $\tilde{E}_{7}$ & $\tilde{E}_{8}$ \\
\hline $\mathbb{Z}_{n}$ & $S O(2)$ & $S O(3)$ & $D_{n}$ & $O(2)$ & $A_{4}$ & $S_{4}$ & $A_{5}$ \\
\hline
\end{tabular}

making correspond graphs and groups having the same algebraic invariants.

Proof. It is convenient to use subgroups of $S U(2)$ rather than subgroups of $S O(3)$. Recall first that we have the following equality:

$$
S U(2)=\left\{\left.\left(\begin{array}{cc}
a & b \\
-\bar{b} & \bar{a}
\end{array}\right)|| a\right|^{2}+|b|^{2}=1\right\}
$$

We regard $a, b$ as complex functions on $S U(2)$, or as elements of $\mathrm{C}(S U(2))$. We also use the real functions $x, y, z, t$ given by $a=x+i y$ and $b=z+i t$.

The change of variables $(a, b) \rightarrow(x, y, z, t)$ gives an isomorphism of real algebraic varieties $S U(2) \simeq S^{3}$. This isomorphism is measure-preserving, in the sense that the Haar measure on $S U(2)$ corresponds to the uniform measure on $S^{3}$.

Now given a subgroup $G \subset S O(3)$, we take its double cover $G^{\prime} \subset S U(2)$. The multiplicity $c_{k}$ for the group $G$, as constructed in the above definition, is

$$
c_{k}=\int \chi^{2 k}=\int(a+\bar{a})^{2 k}=\int\left(4 x^{2}\right)^{k}
$$

Here integration is with respect to the Haar measure of $G^{\prime}$, and we use the functions $\chi, a, x \in \mathrm{C}\left(G^{\prime}\right)$, where $\chi$ is the character of the fundamental representation. 
We can compute now the invariants in the statement.

For $G=S O(3)$ we have $G^{\prime}=S U(2)$. Since $x$ is semicircular on $[-1,1]$, its double $2 x$ is semicircular on $[-2,2]$, so the quadrupled square $4 x^{2}$ is free Poisson on $[0,4]$. We get the measure $\varepsilon=\alpha d$, corresponding to the graph $A_{\infty}$.

For $G=\mathbb{Z}_{n}$ we have $G^{\prime}=\mathbb{Z}_{2 n}$, given in matrix form by:

$$
\mathbb{Z}_{2 n}=\left\{\left(\begin{array}{cc}
q & 0 \\
0 & \bar{q}
\end{array}\right) \mid q^{2 n}=1\right\}
$$

This gives the following formula, where the sum is over $2 n$-roots of unity:

$$
c_{k}=\frac{1}{2 n} \sum_{q}(q+\bar{q})^{2 k}
$$

We get the circular measure $\varepsilon=d_{n}$, corresponding to the graph $\tilde{A}_{2 n-1}$.

For $G=D_{n}$ we have $G^{\prime}=D_{2 n}$, given in matrix form by:

$$
D_{2 n}=\left\{\left(\begin{array}{cc}
q & 0 \\
0 & \bar{q}
\end{array}\right),\left(\begin{array}{cc}
0 & q \\
-\bar{q} & 0
\end{array}\right) \mid q^{2 n}=1\right\}
$$

This gives the following formula, where the sum is over $2 n$-roots of unity:

$$
c_{k}=\frac{1}{4 n} \sum_{q}(q+\bar{q})^{2 k}
$$

We get the circular measure $\varepsilon=\left(d_{1}^{\prime}+d_{n}\right) / 2$, corresponding to the graph $\tilde{D}_{n+2}$.

The limiting cases $S O(2)$ and $O(2)$ are obtained by taking limits.

For $A_{4}, S_{4}, A_{5}$ the situation is a bit more complicated. In lack of a simple direct proof, we prefer to refer here to the McKay correspondence.

Our ADE table is different from the usual one, because it is based on projective representations. For instance the cyclic groups $\mathbb{Z}_{n}$, usually labeled $\tilde{A}_{n}$, are here subject to a rescaling, make them correspond to $\tilde{A}_{2 n-1}$. This is not very surprising, say because of the subfactor point of view, very close to ours: $\tilde{A}_{2 n}$ is known not to be a principal graph. See [24].

We are now in position of finishing the ADE classification of subgroups of $\mathcal{Q}_{4}$. Recall from Theorem 8.1 and Theorem 10.2 that there are a number of subgroups left: 3 infinite series and 5 single quantum subgroups.

Theorem 11.2. We have the classification table

\begin{tabular}{|l|l|l|l|l|l|l|}
\hline$\tilde{A}_{4 n-1}$ & $A_{-\infty, \infty}$ & $A_{\infty}$ & $\tilde{D}_{2 n+2}$ & $D_{\infty}$ & $\tilde{E}_{7}$ & $\tilde{E}_{8}$ \\
\hline$\widehat{D}_{n}$ & $\widehat{D}_{\infty}$ & $\mathcal{Q}_{4}$ & $D_{2 n}^{\tau}, D C_{n}^{\tau}$ & $O_{-1}(2)$ & $S_{4}^{\tau}$ & $A_{5}^{\tau}$ \\
\hline
\end{tabular}

making correspond graphs and quantum groups having the same algebraic invariants.

Proof. It is clear that the algebraic invariants are representation theoretic invariants, and remain the same when passing from a subgroup of $S O(3)$ to its twisted (or pseudo-twisted) version in $S O(3)$. Therefore, for the twists $\mathcal{Q}_{4} \cong S O_{-1}(3), O_{-1}(2)$, $D_{2 n}^{\tau}, D C_{n}^{\tau}, S_{4}^{\tau}, A_{5}^{\tau}$ we can use the previous ADE classification of subgroups of $S O(3)$.

For $\widehat{D}_{n}$ with $n=3,4, \ldots, \infty$ we have to compute the invariants of a certain 4 dimensional representation. But, as for any group dual, this representation must be 
of the form $\pi=\operatorname{diag}\left(g_{i}\right)$, with $D_{n}=<g_{1}, g_{2}, g_{3}, g_{4}>$. Moreover, the multiplicities are given by $c_{k}=\#\left\{\left(i_{1}, \ldots, i_{k}\right) \mid g_{i_{1}} \ldots g_{i_{k}}=1\right\}$. Here we have $D_{n}=\langle 1, g, 1, h\rangle$ where $g^{2}=1=h^{2}$ are the given generators of $D_{n}$ (with the additionnal relation $(g h)^{n}=1$ if $n$ is finite). Thus we have to compute the $k$-loops in the Cayley graph of $D_{n}$ with respect to the set $\{1, g, 1, h\}$, which is $\tilde{A}_{2 n-1}$ with two 1-loops added at each vertex. But this is exactly the number of $2 k$-loops of $\tilde{A}_{4 n-1}$, and this gives the result.

The results in previous sections give the following classification table, where the relation between graphs and subgroups is that the algebraic invariants are the same. The last column contains the idea of the proof.

\begin{tabular}{|l|l|l|}
\hline ADE graph & Subgroup of $\mathcal{Q}_{4}$ & Correspondence \\
\hline$\tilde{A}_{1}, \tilde{A}_{3}, \tilde{A}_{5}, \tilde{A}_{7}$ & $\mathbb{Z}_{1}, \mathbb{Z}_{2}, \mathbb{Z}_{3}, V$ & ADE for $S_{4}$ \\
\hline$\tilde{A}_{4 n-1}$ & $\widehat{D}_{n}$ & Computation \\
\hline$A_{-\infty, \infty}$ & $\widehat{D}_{\infty}$ & Computation \\
\hline$A_{\infty}$ & $\mathcal{Q}_{4}$ & Twisted McKay \\
\hline$\tilde{D}_{4}, \tilde{D}_{6}$ & $\mathbb{Z}_{4}, D_{4}$ & ADE for $S_{4}$ \\
\hline$\tilde{D}_{2 n+2}$ & $D_{2 n}^{\tau}, D C_{n}^{\tau}$ & Twisted McKay \\
\hline$D_{\infty}$ & $O_{-1}(2)$ & Twisted McKay \\
\hline$\tilde{\Delta}_{6}, \tilde{\Delta}_{7}$ & $D_{1}, S_{3}$ & ADE for $S_{4}$ \\
\hline$\tilde{E}_{6}$ & $A_{4}$ & ADE for $S_{4}$ \\
\hline$\tilde{E}_{7}$ & $S_{4}, S_{4}^{\tau}$ & McKay + twist \\
\hline$\tilde{E}_{8}$ & $A_{5}^{\tau}$ & Twisted McKay \\
\hline
\end{tabular}

A second classification table can be obtained by collecting together the ADE classification information for $\mathcal{Q}_{4}$ and for $S O(3)$ (the squares denote missing subgroups):

\begin{tabular}{|l|l|l|}
\hline Subgroup of $S O(3)$ & ADE graph & Subgroup of $\mathcal{Q}_{4}$ \\
\hline $\mathbb{Z}_{1}, \mathbb{Z}_{2}, \mathbb{Z}_{3}, \mathbb{Z}_{4}$ & $\tilde{A}_{1}, \tilde{A}_{3}, \tilde{A}_{5}, \tilde{A}_{7}$ & $\mathbb{Z}_{1}, \mathbb{Z}_{2}, \mathbb{Z}_{3}, V$ \\
\hline $\mathbb{Z}_{2 n-1}, \mathbb{Z}_{2 n}$ & $\tilde{A}_{4 n-3}, \tilde{A}_{4 n-1}$ & $\square, \widehat{D}_{n}$ \\
\hline$S O(2)$ & $A_{-\infty, \infty}$ & $\widehat{D}_{\infty}$ \\
\hline$S O(3)$ & $A_{\infty}$ & $\mathcal{Q}_{4}$ \\
\hline$V, D_{4}$ & $\tilde{D}_{4}, \tilde{D}_{6}$ & $\mathbb{Z}_{4}, D_{4}$ \\
\hline$D_{2 n-1}, D_{2 n}$ & $\tilde{D}_{2 n+1}, \tilde{D}_{2 n+2}$ & $\square, D_{2 n}^{\tau}, D C_{n}^{\tau}$ \\
\hline$O(2)$ & $D_{\infty}$ & $O_{-1}(2)$ \\
\hline$\square, \square$ & $\tilde{\Delta}_{6}, \tilde{\Delta}_{7}$ & $D_{1}, S_{3}$ \\
\hline$A_{4}$ & $\tilde{E}_{6}$ & $A_{4}$ \\
\hline$S_{4}$ & $\tilde{E}_{7}$ & $S_{4}, S_{4}^{\tau}$ \\
\hline$A_{5}$ & $\tilde{E}_{8}$ & $A_{5}^{\tau}$ \\
\hline
\end{tabular}

The following problem appears: is there any "spatial" correspondence between ADE graphs and subgroups of $\mathcal{Q}_{4}$, beyond representation theory results? 
There are several interpretations here:

(1) McKay correspondence. There are many ways of establishing the McKay correspondence for $S O(3)$, see [31]. However, none of them seems to have a clear extension to $\mathcal{Q}_{4}$, or at least we don't know how to find it. One problem here is to find the correct quantum analogue of Platonic solids.

(2) Planar algebras. A statement a bit more "spatial" than the representation theory one can be formulated in terms of planar algebras, by using results in [4]. Thus our ADE classification can be regarded as being part of the ADE classification of index 4 planar algebras, discussed in [25].

(3) Other. There are many other ADE results, for instance Arnold's celebrated classification of singularities [1]. Finding a possible relation with quantum groups seems to be a key problem, that we would like to raise here.

\section{Concluding Remarks}

After Wang's discovery of the quantum permutation group $\mathcal{Q}_{n}$, the classification of its subgroups at $n=4$ given here strengthens the idea that quite unexpected quantum groups might act on classical spaces. In view of Klein's program, this means that a very simple classical space, such as the space consisting of 4 points, might have a very rich quantum geometry.

A natural fundamental question here concerns the existence and construction of Woronowicz' differential calculi [43] on the quantum permutation groups listed in Theorem 1.1. With the exception of the group algebra $\mathbb{C}\left[D_{\infty}\right]$, all the corresponding Hopf algebras are coquasitriangular, so there are well known methods for constructing differential calculi on them. We have two different families:

- The Hopf algebras $\mathcal{R}\left(\mathcal{Q}_{4}\right), \mathcal{R}\left(O_{-1}(2)\right), \mathcal{R}(G)$ for $G \subset S_{4}, \mathcal{R}\left(S_{4}^{\tau}\right), \mathcal{R}\left(A_{5}^{\tau}\right), \mathcal{R}\left(D_{n}^{\tau}\right)$. These Hopf algebras are cotriangular because they are cocycle deformations of function algebras on classical groups. A result of Majid and Oeckl [27] ensures that there is a bijective correspondence between bicovariant differential calculi on these Hopf algebras and on the corresponding function algebras.

- The Hopf algebras $\mathcal{R}\left(D C_{n}^{\tau}\right)$. These are not cotriangular but are coquasitriangular by Suzuki's paper [37]. Therefore we can use the general method of Klimyk and Schmüdgen in chapter 14 of the book [26] for constructing a differential calculus on them.

Concerning the classification problem for quantum permutation groups, it is probably only reachable for small $n$, say $n=5,6,7$, and for general $n$ we only hope to have results splitting the family of quantum subgroups of $\mathcal{Q}_{n}$ in subfamilies having well identified properties. As already said in the introduction, a first obstruction is the complexity of the $\mathrm{C}^{*}$-algebra $\mathrm{C}\left(\mathcal{Q}_{n}\right)$, because of the non-amenability of the discrete quantum group dual to $\mathcal{Q}_{n}[2]$.

A first easy thing to be done is to generalize the usual notions of multiple transitivity to quantum permutation groups, using algebraic invariants as in Section 8. We would like to mention the following question on multiple transitivity. It is known that a classical $k$-transitive permutation group with $k \geq 6$ is either a symmetric or an alternating group. Is there such a kind of result for quantum permutation groups? 
Also it will also be interesting to examine the quantum permutation groups on a prime number of points $p$. Recall that a classical result of Burnside asserts that if $G$ is a subgroup of $S_{p}$ acting transitively on $p$ points, then $G$ is either 2-transitive or is a proper subgroup of the affine group $A G L_{1}\left(\mathbb{Z}_{p}\right)$ [17]. Is there an analogue of Burnside's theorem for quantum permutation groups? A related question was studied in [7].

Finally we have the following conjecture.

Conjecture 12.1. The symmetric group $S_{n}$ is maximal in the quantum permutation group $\mathcal{Q}_{n}$, for any $n$.

As a direct consequence of the classification theorem of quantum subgroups of $\mathcal{Q}_{4}$, the conjecture is true at $n=4$.

\section{REFERENCES}

[1] V.I. Arnold, S.M. Gusein-Zade and A.N. Varchenko, Singularities of differentiable maps, Birkhäuser (1985).

[2] T. Banica, Symmetries of a generic coaction, Math. Ann. 314 (1999), 763-780.

[3] T. Banica, Subfactors associated to compact Kac algebras, Integral Equations Operator Theory 39 (2001), 1-14.

[4] T. Banica, Quantum automorphism groups of homogeneous graphs, J. Funct. Anal. 224 (2005), 243-280.

[5] T. Banica and J. Bichon, Free product formulae for quantum permutation groups, J. Inst. Math. Jussieu 6 (2007), 381-414. .

[6] T. Banica and J. Bichon, Quantum automorphism groups of vertex-transitive graphs of order $\leq 11$, J. Algebraic Combin. 26 (2007), 83-105.

[7] T. Banica, J. Bichon and G. Chenevier, Graphs having no quantum symmetry, Ann. Inst. Fourier 57 (2007), 955-971.

[8] T. Banica, J. Bichon and B. Collins, The hyperoctahedral quantum group, math.RT/0701859.

[9] T. Banica and D. Bisch, Spectral measures of small index principal graphs, Comm. Math. Phys. 269 (2007), 259-281.

[10] T. Banica and B. Collins, Integration over quantum permutation groups, J. Funct. Anal. 242 (2007), 641-657.

[11] T. Banica and B. Collins, Integration over the Pauli quantum group, math.QA/0610041.

[12] T. Banica and S. Moroianu, On the structure of quantum permutation groups, Proc. Amer. Math. Soc. 135 (2007), 21-29.

[13] J. Bichon, Quelques nouvelles déformations du groupe symétrique, C. R. Acad. Sci. Paris 330 (2000), 761-764.

[14] J. Bichon, Quantum automorphism groups of finite graphs, Proc. Amer. Math. Soc. 131 (2003), 665-673.

[15] J. Bichon, Free wreath product by the quantum permutation group, Alg. Rep. Theory 7 (2004), 343-362.

[16] A. Davydov, Galois algebras and monoidal functors between categories of representations of finite groups, J. Algebra 244 (2001), 273-301.

[17] J. Dixon and B. Mortimer, Permutation groups, GTM 163, Springer-Verlag (1996).

[18] Y. Doi, Braided bialgebras and quadratic algebras, Comm. Algebra 21 (1993), 1731-1749.

[19] V. Drinfeld, Quantum groups, Proc. ICM Berkeley (1986), 798-820.

[20] M. Enock and L. Vainerman, Deformation of a Kac algebra by an abelian subgroup, Comm. Math. Phys. 178 (1996), 571-596.

[21] P. Etingof and S. Gelaki, Some properties of finite-dimensional semisimple Hopf algebras, Math. Res. Lett. 5 (1998), 191-197.

[22] P. Etingof and S. Gelaki, The representation theory of cotriangular semisimple Hopf algebras, Internat. Math. Res. Notices 7 (1999), 387-394. 
[23] P. Etingof and S. Gelaki, The classification of triangular semisimple and cosemisimple Hopf algebras over an algebraically closed field, Internat. Math. Res. Notices 5 (2000), 223-234.

[24] D. Evans and Y. Kawahigashi, Quantum symmetries on operator algebras, Oxford University Press (1998).

[25] V.F.R. Jones, The annular structure of subfactors, Monogr. Enseign. Math. 38 (2001), 401-463.

[26] A. Klimyk and K. Schmüdgen, Quantum groups and their representations, Texts and Monographs in Physics, Springer-Verlag, Berlin (1997).

[27] S. Majid and R. Oeckl, Twisting of quantum differentials and the Planck scale Hopf algebra, Comm. Math. Phys. 205 (1999), 617-655.

[28] Y. Manin, Quantum groups and noncommutative geometry, Publications du CRM 1561, Univ. de Montréal (1988).

[29] V.A. Marchenko and L.A. Pastur, Distribution of eigenvalues in certain sets of random matrices, Mat. Sb. 72 (1967), 507-536.

[30] A. Masuoka, Cocycle deformations and Galois objects for some cosemisimple Hopf algebras of finite dimension, Contemp. Math. 267 (2000), 195-214.

[31] J. McKay, Graphs, singularities and finite groups, Proc. Symp. Pure Math. 37 (1980), 183-186.

[32] W.D. Nichols and M.B. Zoeller, A Hopf algebra freeness theorem, Amer. J. Math. 111 (1989), 381-385.

[33] D. Nikshych, $K_{0}$-rings and twisting of finite-dimensional semisimple Hopf algebras, Comm. Algebra 26 (1998), 321-342.

[34] P. Podles, Symmetries of quantum spaces. Subgroups and quotient spaces of quantum SU(2) and SO(3) groups, Comm. Math. Phys. 170 (1995), 1-20.

[35] M. Rosso, Algèbres enveloppantes quantifiées, groupes quantiques compacts de matrices et calcul differentiel non-commutatif, Duke Math. J. 61 (1990), 11-40.

[36] P. Schauenburg, Hopf bigalois extensions, Comm. Algebra 24 (1996), 3797-3825.

[37] S. Suzuki, A family of braided cosemisimple Hopf algebras of finite dimension, Tsukuba J. Math. 22 (1998), 1-29.

[38] L. Vainerman, 2-cocycles and twisting of Kac algebras, Comm. Math. Phys. 191 (1998), 697721.

[39] D.V. Voiculescu, K.J. Dykema and A. Nica, Free random variables, CRM Monograph Series 1, AMS (1992).

[40] S. Wang, Free products of compact quantum groups, Comm. Math. Phys. 167 (1995), 671-692.

[41] S. Wang, Quantum symmetry groups of finite spaces, Comm. Math. Phys. 195 (1998), 195-211.

[42] S.L. Woronowicz, Compact matrix pseudogroups, Comm. Math. Phys. 111 (1987), 613-665.

[43] S.L. Woronowicz, Differential calculus on compact matrix pseudogroups (quantum groups), Comm. Math. Phys. 122 (1989), 125-170.

[44] S.L. Woronowicz, Compact quantum groups, in "Symétries quantiques" (Les Houches, 1995), North Holland, Amsterdam (1998), 845-884.

[45] S. Zakrzewski, Matrix pseudogroups associated with anti-commutative plane, Lett. Math. Phys. 21 (1991), 309-321.

Laboratoire de Mathématiques, Université Paul Sabatier, Toulouse III, 118 route DE NARbonne, 31062 Toulouse, France

E-mail address: Teodor.Banica@math.ups-tlse.fr

Laboratoire de Mathématiques, Université Blaise Pascal, Clermont-Ferrand II, Campus des Cézeaux, 63177 Aubière Cedex, France

E-mail address: Julien.Bichon@math.univ-bpclermont.fr 